\title{
Los patelogastrópodos intermareales de Chile y Perú
}

\author{
Intertidal limpets of Chile and Perú
}

CARMEN ESPOZ ${ }^{1,3}$, DAVID R. LINDBERG ${ }^{2}$, JUAN C. CASTILLA ${ }^{1} \&$ W. BRIAN SIMISON ${ }^{2}$

\author{
${ }^{1}$ Departamento de Ecología, Facultad de Ciencias Biológicas, \\ Pontificia Universidad Católica de Chile, Santiago, Chile \\ ${ }^{2}$ Museum of Paleontology, University of California, Berkeley, California, USA \\ ${ }^{3}$ Dirección actual: Departamento de Ciencias Básicas, Universidad Santo Tomás, Ejército 146, Santiago, Chile; \\ e-mail: cespoz@ust.cl
}

\begin{abstract}
RESUMEN
En este estudio se entrega un sistema de clasificación que refleja las relaciones de parentesco, inferidas a partir del análisis de la región $16 \mathrm{~S}$ de ADN mitocondrial, de los patelogastrópodos que habitan en la zona intermareal a lo largo de la costa rocosa de Chile y Perú. Además, se incluye el análisis comparativo de estos patelogastrópodos en relación con la estructura, la morfología y los patrones de color de la concha, los dientes de la rádula y la anatomía corporal. Adicionalmente, se entrega información respecto de la distribución y ecología para todos los miembros de este grupo. Los resultados muestran que la fauna Lottiidae de Chile y Perú consiste en al menos nueve especies de patelogastrópodos intermareales. Esto es, ocho especies de patelogastrópodos agrupadas dentro del clado monofilético Scurria (S. variabilis, S. zebrina, S. viridula, S. plana, S. scurra, S. araucana, S. ceciliana y una especie no determinada) y un "taxón problemático" asignado preliminarmente dentro del género Lottia ('Lottia' orbignyi). El grupo está presente entre los $5^{\circ} \mathrm{S}$ y $54^{\circ} \mathrm{S}$, registrándose en los niveles intermareales superior, medio e inferior, desde zonas expuestas a protegidas del oleaje. A nivel intraespecífico, los resultados nos permiten proponer la sinonimia de $S$. parasitica con $S$. variabilis, y de $S$. ceciliana con $S$. boehmita. Según esto, $S$. variabilis presentaría al menos tres ecofenotipos: (1) en sustratos rocosos a lo largo de todo su rango geográfico, (2) sobre las conchas de Fissurella crassa y $F$. limbata principalmente en Chile centro-sur y (3) sobre las placas del chitón Enoplochiton niger en el norte de Chile y sur del Perú. Por su parte, S. ceciliana es una especie de patelogastrópodo que presenta un patrón morfológico y de coloración de la concha altamente variable. En general, la alta variabilidad del patrón de color y morfología de la concha sitúa a estos patelogastrópodos entre los más complejos y diversos del mundo. Más aún, esta variabilidad y flexibilidad pueden ser responsables de las confusiones taxonómicas que han rodeado a este conjunto faunístico.
\end{abstract}

Palabras clave: Patellogastropoda, intermareal rocoso, Chile, Perú, filogenia, ecología.

\begin{abstract}
In this study we present a classification system reflecting the phylogenetic relationships for the intertidal patellogastropods of Chile and Perú. We infer phylogenetic relationships between limpets species using DNA sequences from the $16 \mathrm{~S}$ region of the mitochondrial genome. We include a comparative limpet study of: shell structure, color and morphological patterns of the shell, radular teeth and anatomy. Additionally, we provide information about the distribution and ecology for members of this group within the studied area. The results show a Chilean-Peruvian Lottiidae fauna consisting of at least nine species, which are grouped into a single monophyletic clade Scurria (S. variabilis, S. zebrina, S. viridula, S. plana, S. scurra, S. araucana, S. ceciliana and a non identificated species), and a "problematic taxon" preliminarily assigned to the genus Lottia ('Lottia' orbignyi). The group of species is present in the intertidal rocky shores between $5^{\circ} \mathrm{S}$ and $54^{\circ} \mathrm{S}$, from the upper to the lower intertidal fringes, ranging from exposed to protected areas. The systematic outcome of this study suggests the synonymization of $S$. parasitica to $S$. variabilis, and of S. ceciliana to $S$. boehmita. According to our field results, the species $S$. variabilis shows at least three ecophenotypes: (1) on rocky substrates along the full geographic range, (2) on the shells of the key-hole limpets: Fissurella crassa and F. limbata mainly in central and south of Chile, and (3) on the chiton plates of Enoplochiton niger in the north of Chile and south of Perú. S. ceciliana is a species in which morphology and color patterns are highly variable. In general, this variability indicates that the Chilean-Peruvian limpet species are among the most complex and diverse limpet fauna in the world. This, may reflects the taxonomic confusion which has beset this fauna.
\end{abstract}

Key words: Patellogastropoda, limpets, rocky intertidal, Chile, Perú, phylogeny, ecology. 


\section{INTRODUCCIÓN}

El orden Patellogastropoda (Mollusca) agrupa a aquellas especies que pertenecen a las familias Patellidae, Nacellidae y Lottidae, las últimas previamente referidas como Acmaeidae (Lindberg 1998). Originalmente, el grupo de los patelogastrópodos fue asignado a la subclase Archaeogastropoda. Sin embargo, a partir del estudio realizado por Golikov \& Starobogatov (1975) este fue reasignado al orden Docoglossa. En 1988, Lindberg (1988a, 1988b) recomienda la creación de un nuevo orden denominado Patellogastropoda diferente del orden Docoglossa dado que este último incluye un tipo de morfología radular, muscular y de funcionamiento que no es única a los patelogastrópodos. Así, los patelogastrópodos son definidos como un orden separado dentro de los gastrópodos prosobranquiados (Lindberg 1988a, 1998), debido al reconocimiento de una línea evolutivamente distinta del resto de los gastrópodos, lo que ha sido corroborado por Ponder \& Lindberg (1997), Lindberg (1998) y Koufopanou et al. (1999).

A nivel mundial, los patelogastrópodos son reconocidos como miembros abundantes y ecológicamente importantes de la mayoría de las comunidades marinas (Branch 1985a, 1985b, Lindberg 1988a, 1998). En Chile y Perú, los patelogastrópodos están representados por numerosas especies todas las cuales son miembros comunes y frecuentes de las comunidades intermareales rocosas entre los $4^{\circ} \mathrm{S}$ y $54^{\circ} \mathrm{S}$ (Dall 1909, Marincovich 1973, Paredes 1980, Castilla 1981), a pesar de lo cual la información ecológica existente es escasa (como excepciones ver Hockey et al. 1987, Muñoz \& Santelices 1989, Espoz et al. 1995, Espoz \& Castilla 2000, Espoz 2002). No obstante lo anterior, la ocurrencia del grupo a lo largo del margen costerolitoral de Chile y Perú aparece bien documentada. Entre los autores que han estudiado los patelogastrópodos endémicos de esta costa se encuentran Pilsbry (1891), Dall (1909), Thiem (1917), MacClintock (1967), Marincovich (1973), Paredes (1980) y Alamo \& Valdivieso (1987), quienes los incorporan dentro de revisiones taxonómicas mayores que abarcan al conjunto de moluscos registrados dentro de la Provincia Biogeográfica Peruana (sensu Dall 1909). También existen estudios sobre el grupo que se centran en áreas geográficas más restringidas como son la costa rocosa del Departamento de Lima (Perú) (Paredes 1974), la Provincia de Islay (Arequipa, Perú) (Talledo \& González 1989) o la zona intermareal de Papu- do (Chile) (Ramírez 1974). En la Tabla 1 se presenta el sistema de clasificación propuesto por Marincovich (1973), quien resume la información disponible para el grupo. El autor agrupa a los patelogastrópodos que habitan en la zona intermareal rocosa de Chile y Perú dentro de la familia Acmaeidae, actualmente reconocida como familia Lottiidae (Lindberg 1998) y los asigna a dos géneros, Collisella y Scurria, dependiendo de la presencia o ausencia de cordón branquial. Sin embargo, debido a que los patelogastrópodos chileno-peruanos muestran un desarrollo parcial del cordón branquial, el autor indica que esta asignación es arbitraria y debe ser revisada en el futuro. En cuanto a la asignación de especies, Marincovich (1973) se basó principalmente en la morfología y los patrones de color de la concha. Si bien en este grupo la morfología de la concha suele ser diagnóstica al nivel de especie, esta debe ser utilizada con precaución cuando se infieren las relaciones entre los miembros de la familia Lottiidae puesto que en la familia estas morfologías presentan altos grados de convergencia y paralelismo (Lindberg 1988b). Así, numerosas especies de esta familia han evolucionado hacia morfologías de concha semejantes, a pesar que son miembros de clados diferentes (Lindberg 1988b).

\section{TABLA 1}

Resumen de la clasificación existente para los patelogastrópodos intermareales de la familia Lottiidae presentes a lo largo de la costa rocosa de Chile y Perú. El esquema corresponde al publicado por Marincovich (1973) quien resume la información disponible para el grupo

Summary of the current available classification information for rocky intertidal limpets, family Lottiidae, documented for Chile and Perú (taken from Marincovich 1973)

Clasificación propuesta por Marincovich (1973)

Familia Acmaeidae (Forbes, 1850)

Collisella (Dall, 1871)

Collisella araucana (Orbignyi, 1839)

Collisella ceciliana (Orbignyi, 1841)

Collisella orbignyi (Dall, 1909)

Collisella variabilis (Sowerby, 1839)

Collisella zebrina (Lesson, 1830)

Scurria (Gray, 1847)

Scurria parasitica (Orbignyi, 1841)

Scurria scurra (Lesson, 1830)

Scurria viridula (Lamarck, 1819) 
Idealmente, cada unidad de clasificación debiera corresponder a una rama evolutiva o clado (Vermeij 1993). Un clado tiene un solo ancestro común y contiene a todos sus descendientes. La unidad se distingue por características no compartidas con otros clados, pudiendo estar evolutivamente relacionada con otro clado por caracteres que ambos clados tienen en común, en virtud de un ancestro común. De acuerdo con esto, debe esperarse que las categorías dentro del esquema de clasificación actualmente aplicado para los patelogastrópodos, cumplan con estos criterios. Los únicos estudios donde se considera el análisis filogenético de patelogastrópodos que habitan a lo largo del margen costero del Pacífico este son los de $\mathrm{Si}$ mison (2000) y Espoz (2002). Aun cuando el estudio de Simison (2000) no tuvo como objetivo la revisión sistemática del grupo, el análisis permitió establecer la monofilia del grupo de patelogastrópodos chileno-peruanos, siendo esta la única información filogenética disponible para el mencionado grupo de patelogastrópodos.

En función de estos antecedentes, el objetivo de este estudio es establecer un marco de referencia sistemático y taxonómico básico para estudios futuros de distribución y abundancia de patelogastrópodos intermareales endémicos de Chile y Perú. Específicamente, se estiman las relaciones de parentesco a través del análisis de caracteres moleculares y se propone un sistema de clasificación que refleja estas relaciones.

\section{METODOLOGÍA}

\section{Selección y recolección de taxa}

De acuerdo con la información obtenida en el terreno y después del análisis de las colecciones de patelogastrópodos de diferentes museos (ver más adelante) se hizo una selección de todas las morfologías que fue posible distinguir, referidas a la apariencia y al hábitat donde fueron registrados los individuos. De cada una de estas formas se seleccionó un representante lo que se tradujo en un total de 17 muestras a analizar. Estas muestras incluyeron: (1) especies taxonómicamente establecidas, (2) ecofenotipos (i.e., morfologías específicas y patrones de color asociados con hábitats particulares) (Lindberg 1981, Sorensen \& Lindberg 1991), y (3) taxa únicos en diferentes regiones geográficas. Los especímenes fueron obtenidos en recolecciones realizadas entre los meses de febrero y diciembre de 1998 en los si- guientes sitios: (1) San Pablo, Departamento de Moquegua, Perú (1800' S; 7053' O), (2) Algarrobo, Quinta Región, Chile (33⒉ $1^{\prime}$ S; $71^{\circ} 40^{\prime}$ O), (3) Las Cruces, Quinta Región,

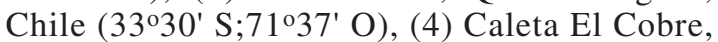
Segunda Región, Chile (24ํํ' S; 70³2' O), (5) Guanillo, Segunda Región, Chile (255' S; 7041' O), (6) Pelancura, Quinta Región,

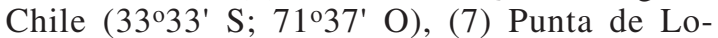
bos, Sexta Región, Chile ( $\left.34^{\circ} 25^{\prime} \mathrm{S} ; 7^{\circ} 02^{\prime} \mathrm{O}\right)$ y (8) Pucatrihue, Décima Región, Chile (4032' S; 734' O). Una vez recolectados, los ejemplares fueron preservados en Etanol al 70 $\%$. Todo el material recolectado y analizado en el marco de este estudio fue depositado en el Museo de Paleontología, Universidad de California, Berkeley, Estados Unidos.

\section{Extracción de ADN}

Para detectar divergencia molecular entre especies de patelogastrópodos se secuenció la región $16 \mathrm{~S}$ de ADN mitocondrial (Simison 2000). Para aislar el ADN se siguió el protocolo de extracción CTAB/PHENOL descrito por Palumbi et al. (1991). El procedimiento consiste en cortar una porción de tejido pedal (3-5 mm de diámetro). Posteriormente, el tejido se lava con agua destilada y se corta finamente. Estos trozos de tejido se sumergen en $600 \mathrm{ml}$ de 2 XCTAB y $9 \mathrm{ml}$ de proteinasa $\mathrm{K}$. La solución se incuba a $37{ }^{\circ} \mathrm{C}$ durante $12 \mathrm{~h}$. Después de agregar $600 \mathrm{ml}$ de fenol: cloroformo: alcoholisoamil (25:24:1), la solución se centrifuga a $13 \mathrm{k}$ por $15 \mathrm{~min}$. El sobrenadante se mezcla con $600 \mathrm{ml}$ de cloroformo:alcoholisoamil (24:1) por 5 min y luego se centrifuga a $13 \mathrm{k}$ por $15 \mathrm{~min}$. El ADN es precipitado, usando $600 \mathrm{ml}$ de isopropanol y almacenado a $-20{ }^{\circ} \mathrm{C}$ por $2 \mathrm{~h}$. El precipitado se centrifuga a $13 \mathrm{k}$ por $30 \mathrm{~min}$ a $4{ }^{\circ} \mathrm{C}$. El sobrenadante se elimina y el pellet se lava dos veces con dos volúmenes de Etanol al $70 \%$. Posteriormente, se centrifuga a $13 \mathrm{k}$ por $20 \mathrm{~min}$ y se elimina el Etanol. El pellet se seca al vacío por $5 \mathrm{~min}$, a lo cual sigue una resuspensión en $100 \mathrm{ml}$ de agua destilada.

\section{Amplificación del gen $16 \mathrm{~S}$}

La amplificación del gen mitocondrial $16 \mathrm{~S}$ se hizo con los partidores 16 Sar y 16 Sbr (Palumbi 1996). En un tubo de $0.5 \mathrm{ml}$ colocado sobre hielo se mezcló: $36.45 \mathrm{ml}$ de agua doblemente destilada, $5 \mathrm{ml}$ de 10x buffer de PCR (Perkin Elmer), $2.5 \mathrm{ml}$ de $10 \mathrm{mM}$ dNTP's (Pharmacia), $2.5 \mathrm{ml}$ de $25 \mathrm{mM} \mathrm{MgCl}{ }_{2}$ (Perkin Elmer), $1 \mathrm{ml}$ de $10 \mathrm{mM}$ de los dos partidores respecti- 
vamente, $1 \mathrm{ml}$ de templado y $0.25 \mathrm{ml}$ de polimerasa Taq (Perkin Elmer). En paralelo se amplificó un control negativo con todos los agentes excepto el templado. Luego, el tubo fue transferido a un termociclador Perkin Elmer 9.600. Los parámetros de ciclado comenzaron con una denaturalización a $95{ }^{\circ} \mathrm{C}$ por 2 min seguido de 36 ciclos con tres máximos de temperaturas: $95{ }^{\circ} \mathrm{C}$ por $50 \mathrm{~s}, 45^{\circ} \mathrm{C}$ por $50 \mathrm{~s} \mathrm{y}$ $72{ }^{\circ} \mathrm{C}$ por $90 \mathrm{~s}$, terminando con una extensión de 7 min a $72{ }^{\circ} \mathrm{C}$. Los productos PCR fueron purificados usando un sistema de purificación de ADN Wizard.

\section{Secuenciación y alineación}

La secuenciación cíclica de doble hebra directa de 20 a $30 \mathrm{ng}$ de producto purificado del gen $16 \mathrm{~S}$ se hizo en ambas direcciones usando un equipo para secuenciación cíclica $\mathrm{ABI}$. Para la secuenciación cíclica se utilizó un termociclador Perkin Elmer 9.600. Los parámetros de ciclado fueron de 25 ciclos a: $96{ }^{\circ} \mathrm{C}$ por $10 \mathrm{~s}, 50{ }^{\circ} \mathrm{C}$ por $5 \mathrm{~s}$ y $60{ }^{\circ} \mathrm{C}$ por $4 \mathrm{~min}$. El producto de secuenciación cíclica fue purificado, secado en una centrífuga al vacío y finalmente fue resuspendido con $2.5 \mathrm{ml}$ de solución de carga de 5:1 de formamida: $25 \mathrm{mM}$ deionizada EDTA con $50 \mathrm{mg} \mathrm{ml}^{-1}$ dextran azul. $1.5 \mathrm{ml}$ de muestra. La solución de carga fue puesta sobre un gel de $36 \mathrm{~cm}$ al $4 \%$ de acrilamida. El gel fue corrido y analizado sobre un secuenciador ABI Prism ${ }^{\circledR}, 377$. Una vez obtenidas las secuencias se procedió con la alineación de estas. Todas las secuencias fueron alineadas a mano usando un ABI, Sequence Navigator.

\section{Inferencia filogenética}

El análisis de los datos moleculares se hizo de acuerdo con los principios de la sistemática filogenética, específicamente bajo la concepción cladista (Wiley 1981, Brooks \& McLennan 1991, Wiley et al. 1991). En este contexto, la polarización de los caracteres fue realizada por comparación con grupos externos (Wiley 1981, Maddison et al. 1984, Lindberg 1991, Wiley et al. 1991). El grupo externo correspondió a los patelogastrópodos de la familia Lottiidae presentes en California y el Caribe. El análisis filogenético de los datos fue realizado mediante el uso del programa computacional PAUP 4.0* (Swofford 1999). Se trabajó con los criterios de optimización de parsimonia y máxima probabilidad. En ambos casos se realizó una búsqueda heurística con una adición de secuencias del tipo ASIS. El algoritmo usado para el procedimiento de intercambio de ramas "branch swapping" fue el denominado TBR "Tree Bisection Reconnection". La obtención de niveles de confianza se hizo mediante técnicas de remuestreo "boostrapping" (Manly 1986), el cual se aplicó tanto al análisis de parsimonia como al de máxima verosimilitud. En ambos casos se utilizaron 1.000 réplicas. Como medida del grado de parentesco entre pares de especies de patelogastrópodos se utilizaron la distancia genética expresada como la distancia absoluta que corresponde al número total de pares de bases diferentes, el que se expresa como el porcentaje en relación al total de sitios disponibles. Este análisis también fue realizado considerando la distancia calculada según el modelo de dos parámetros (Kimura 1980). Las distancias medias entre pares de especies dentro y entre clados fueron calculadas manualmente a partir de las matrices de distancia producidas por el programa Paup 4.0* (Swofford 1999).

\section{Análisis comparativo}

El análisis comparativo de la estructura y morfología de la concha, dientes de la rádula, aspectos específicos de la anatomía corporal y los patrones de color de la concha que se entregan en la descripción de cada especie de patelogastrópodo se basaron en la revisión de las colecciones sistemáticas de: (1) Colección de Flora y Fauna Profesor Patricio Sánchez Reyes de la Pontificia Universidad Católica de Chile (Santiago, Chile), (2) Museo de Zoología de la Universidad de Concepción (Concepción, Chile), (3) Museo Nacional de Historia Natural (Santiago, Chile) y (4) Museo de Paleontología de la Universidad de California (Berkeley, Estados Unidos). A su vez, este análisis comparativo permitió establecer las sinonimias de especies que se presentan junto a la descripción de cada especie. La información ecológica y la distribución geográfica que se entrega para cada especie de patelogastrópodo se basó en el muestreo de 54 sitios (Tabla 2) ubicados a lo largo de la costa rocosa de Chile y Perú, entre los $3^{\circ} \mathrm{S}$ y $54^{\circ} \mathrm{S}$, y corresponde a antecedentes entregados por Espoz (2002).

\section{RESULTADOS}

\section{Filogenia}

De las 17 secuencias moleculares analizadas, ocho mostraron un rango de distancias absolutas que fluctuó entre 0 y 9 pares de bases dis- 


\section{TABLA 2}

Lista de los sitios muestreados entre los meses de julio de 1997 y febrero de 1999 a lo largo de la costa rocosa de Chile y Perú.

La distancia lineal entre los sitios extremos correspondió a 5.800 km (extraída de Espoz 2002)

Sites sampled between July 1997 and February 1999 along the rocky shore of Chile and Perú. Lineal distance between the extreme sites was $5,800 \mathrm{~km}$ (taken from Espoz 2002)

\begin{tabular}{|c|c|c|}
\hline Localidad & $\begin{array}{l}\text { Latitud } \\
{ }^{\circ} \mathrm{S}\end{array}$ & $\begin{array}{c}\text { Longitud } \\
{ }^{\circ} \mathrm{O}\end{array}$ \\
\hline Zorritos & $03^{\circ} 40^{\prime} 45^{\prime \prime}$ & $80^{\circ} 40^{\prime} 20^{\prime \prime}$ \\
\hline Punta Mero & $03^{\circ} 54^{\prime} 01^{\prime \prime}$ & $80^{\circ} 52^{\prime} 25^{\prime \prime}$ \\
\hline Máncora & $04^{\circ} 07^{\prime} 04^{\prime \prime}$ & $81^{\circ} 05^{\prime} 13^{\prime \prime}$ \\
\hline Los Cangrejos, Paita & $05^{\circ} 08^{\prime} 40^{\prime \prime}$ & $81^{\circ} 10^{\prime} 27^{\prime \prime}$ \\
\hline Punta Salinas, El Huacho & $11^{\circ} 09^{\prime} 07^{\prime \prime}$ & $77^{\circ} 36^{\prime} 11^{\prime \prime}$ \\
\hline Naplo, Pucusana & $12^{\circ} 28^{\prime} 26^{\prime \prime}$ & $76^{\circ} 47^{\prime} 33^{\prime \prime}$ \\
\hline Mendieta, Paracas & $14^{\circ} 03^{\prime} 46^{\prime \prime}$ & $76^{\circ} 16^{\prime} 12^{\prime \prime}$ \\
\hline San Juan de Marcona & $15^{\circ} 21^{\prime} 22^{\prime \prime}$ & $75^{\circ} 11^{\prime} 04^{\prime \prime}$ \\
\hline Atico & $16^{\circ} 13^{\prime} 32^{\prime \prime}$ & $73^{\circ} 40^{\prime} 10^{\prime \prime}$ \\
\hline La Ballenita, Mollendo & $17^{\circ} 00^{\prime} 5$ & $72^{\circ} 02^{\prime} 22^{\prime \prime}$ \\
\hline San Pablo, Ilo & $8^{\circ} 00^{\prime} 2$ & $70^{\circ} 53^{\prime} 07^{\prime \prime}$ \\
\hline Arica & $8^{\circ} 31^{\prime} 1$ & $70^{\circ} 19^{\prime} 12^{\prime \prime}$ \\
\hline Camarones & $9^{\circ} 12^{\prime}$ & $70^{\circ} 16^{\prime} 07^{\prime \prime}$ \\
\hline Pisa & $9^{\circ} 3$ & $0^{\circ} 12^{\prime} 19^{\prime \prime}$ \\
\hline erdes, Iquique & $0^{\circ} 25^{\prime}$ & $0^{\circ} 09^{\prime} 56^{\prime \prime}$ \\
\hline Punta & $0^{\circ} 51^{\prime} 3$ & $0^{\circ} 09^{\prime} 17^{\prime \prime}$ \\
\hline Chipa & $1^{\circ} 15^{\prime}$ & $56^{\prime \prime}$ \\
\hline Tocop & $1^{\circ} 5$ & $43^{\prime \prime}$ \\
\hline a-Guala & $2^{\circ} 4$ & $38^{\prime \prime}$ \\
\hline asta & $3^{\circ} 3$ & $0^{\circ} 32^{\prime} 02^{\prime \prime}$ \\
\hline Cobre & $24^{\circ} 19^{\prime} 22^{\prime \prime}$ & $70^{\circ} 32^{\prime} 30^{\prime \prime}$ \\
\hline a, Taltal & $25^{\circ} 11^{\prime} 39^{\prime \prime}$ & $70^{\circ} 26^{\prime} 06^{\prime \prime}$ \\
\hline has, Taltal & $25^{\circ} 18^{\prime} 25^{\prime \prime}$ & $70^{\circ} 27^{\prime} 10^{\prime \prime}$ \\
\hline Guan & $25^{\circ} 53^{\prime} 44^{\prime \prime}$ & $70^{\circ} 41^{\prime} 28^{\prime \prime}$ \\
\hline del Inca & $26^{\circ} 36^{\prime} 29^{\prime \prime}$ & $70^{\circ} 42^{\prime} 27^{\prime \prime}$ \\
\hline Viejo & $27^{\circ} 22^{\prime} 14^{\prime \prime}$ & $70^{\circ} 57^{\prime} 37^{\prime \prime}$ \\
\hline ualtatas, Huasco & $28^{\circ} 12^{\prime} 07^{\prime \prime}$ & $71^{\circ} 09^{\prime} 38^{\prime \prime}$ \\
\hline Choros Norte & $29^{\circ} 06^{\prime} 49^{\prime \prime}$ & $71^{\circ} 28^{\prime} 14^{\prime \prime}$ \\
\hline Caleta Hornos & $29^{\circ} 38^{\prime} 39^{\prime \prime}$ & $71^{\circ} 18^{\prime} 41^{\prime \prime}$ \\
\hline Puerto Aldea, Tongoy & $30^{\circ} 17^{\prime} 14^{\prime \prime}$ & $71^{\circ} 36^{\prime} 36^{\prime \prime}$ \\
\hline Caleta Derrumbe & $31^{\circ} 12^{\prime} 53^{\prime \prime}$ & $71^{\circ} 38^{\prime} 34^{\prime \prime}$ \\
\hline Los Vilos & $31^{\circ} 55^{\prime} 40^{\prime \prime}$ & $71^{\circ} 31^{\prime} 09^{\prime \prime}$ \\
\hline Zapallar & $32^{\circ} 29^{\prime} 57^{\prime \prime}$ & $71^{\circ} 27^{\prime} 52^{\prime \prime}$ \\
\hline Laguna Verde, Valparaíso & $33^{\circ} 05^{\prime} 27^{\prime \prime}$ & $71^{\circ} 43^{\prime} 38^{\prime \prime}$ \\
\hline El Quisco & $33^{\circ} 23^{\prime} 45^{\prime \prime}$ & $71^{\circ} 42^{\prime} 13^{\prime \prime}$ \\
\hline Pelancura & $33^{\circ} 33^{\prime} 18^{\prime \prime}$ & $71^{\circ} 37^{\prime} 39^{\prime \prime}$ \\
\hline Matanzas & $33^{\circ} 57^{\prime} 54^{\prime \prime}$ & $71^{\circ} 53^{\prime} 02^{\prime \prime}$ \\
\hline Punta de Lobos, Pichilemu & $34^{\circ} 25^{\prime} 40^{\prime \prime}$ & $72^{\circ} 02^{\prime} 57^{\prime \prime}$ \\
\hline El Duao, Pichilemu & $34^{\circ} 53^{\prime} 34^{\prime \prime}$ & $72^{\circ} 10^{\prime} 36^{\prime \prime}$ \\
\hline Constitución & $35^{\circ} 21^{\prime} 35^{\prime \prime}$ & $71^{\circ} 27^{\prime} 58^{\prime \prime}$ \\
\hline Pelluhue & $35^{\circ} 49^{\prime} 25^{\prime \prime}$ & $72^{\circ} 36^{\prime} 27^{\prime \prime}$ \\
\hline Buchupureo & $36^{\circ} 04^{\prime} 47^{\prime \prime}$ & $72^{\circ} 48^{\prime} 28^{\prime \prime}$ \\
\hline Cocholgue, Concepción & $36^{\circ} 36^{\prime} 42^{\prime \prime}$ & $72^{\circ} 58^{\prime} 38^{\prime \prime}$ \\
\hline Laraquete & $37^{\circ} 09^{\prime} 52^{\prime \prime}$ & $73^{\circ} 11^{\prime} 26^{\prime \prime}$ \\
\hline Lebu Norte & $37^{\circ} 34^{\prime} 44^{\prime \prime}$ & $73^{\circ} 38^{\prime} 34^{\prime \prime}$ \\
\hline Quidico & $38^{\circ} 15^{\prime} 12^{\prime \prime}$ & $73^{\circ} 30^{\prime} 22^{\prime \prime}$ \\
\hline Yupehue Bajo & $38^{\circ} 32^{\prime} 25^{\prime \prime}$ & $73^{\circ} 30^{\prime} 18^{\prime \prime}$ \\
\hline Mehuín & $39^{\circ} 25^{\prime} 14^{\prime \prime}$ & $73^{\circ} 13^{\prime} 07^{\prime \prime}$ \\
\hline Pucatrihue, Osorno & $40^{\circ} 32^{\prime} 51^{\prime \prime}$ & $73^{\circ} 43^{\prime} 09^{\prime \prime}$ \\
\hline Chepu, Chiloé & $42^{\circ} 02^{\prime} 40^{\prime \prime}$ & $74^{\circ} 08^{\prime} 05^{\prime \prime}$ \\
\hline Huentemó, Chiloé & $42^{\circ} 32^{\prime} 16^{\prime \prime}$ & $74^{\circ} 02^{\prime} 03^{\prime \prime}$ \\
\hline Pirulil, Chiloé & $42^{\circ} 42^{\prime} 25^{\prime \prime}$ & $74^{\circ} 09^{\prime} 23^{\prime \prime}$ \\
\hline Fuerte Bulnes, Punta Arenas & $53^{\circ} 37^{\prime} 39^{\prime \prime}$ & $70^{\circ} 55^{\prime} 06^{\prime \prime}$ \\
\hline Isla Navarino & $54^{\circ} 54^{\prime} 35^{\prime \prime}$ & $67^{\circ} 49^{\prime} 37^{\prime \prime}$ \\
\hline
\end{tabular}

tintas por 724 sitios (0-1,2\%) (Tabla 3), lo cual se ubica dentro del rango de variación registrado entre individuos de una misma especie de patelogastrópodo (Simison \& Lindberg 1999). De acuerdo con los resultados (ver Tabla 3 para nomenclatura utilizada), Scurria variabilis reúne a los patelogastrópodos previamente nominados como $S$. parasitica, incluidos los ejemplares que habitan sobre las conchas de lapas del género Fissurella ( $S$. parasitica-Fiss) y sobre las placas de Enoplochiton niger (S. parasitica-En). Asimismo, S. variabilis incluye a dos formas transicionales registradas entre los $25^{\circ} \mathrm{S}-28^{\circ} \mathrm{S}$ (SVSP1) y otra en Pelancura en Chile central $\left(33^{\circ} \mathrm{S}\right)$ (SVSP2). Los resultados muestran que $S$. araucana presenta una forma transicional que habita en pozas intermareales, principalmente en la zona central de Chile (PO). En cambio, $S$. scurra aparece con una forma registrada sobre las valvas del mitílido Perumytilus purpuratus (CP2). Por último, S. boehmita queda incluida en $S$. ceciliana, al igual que una forma que vive sobre $P$. purpuratus (CP1). A nivel de diferencias entre conespecíficos y la ocurrencia de transiciones/transversiones (Tabla $4)$, los resultados indican que estas fueron solo de 0-2 transiciones sin registrarse diferencias en el número de transversiones, salvo en los casos del par formado por S. araucana y la forma que habita en pozas (PO) donde las diferencias fueron de dos transiciones y dos transversiones, y el par formado por S. scurra y una forma registrada sobre $P$. purpuratus (CP3) con 9 transiciones de diferencia.

A nivel interespecífico, las distancias variaron entre 15-155 pares de bases por 724 sitios $(2,1-21,4 \%)$ (Tabla 3), con lo cual se definió la existencia de 9 especies de patelogastrópodos. Esto es, siete especies de patelogastrópodos agrupadas dentro de un clado que fueron asignadas al género Scurria: S. variabilis, S. zebrina, S. viridula, S. plana, S. scurra, S. araucana, S. ceciliana; un taxón problemático (ver discusión más adelante) asignado preliminarmente dentro del género Lottia ('Lottia' orbignyi) y una especie identificada a nivel de género (CP3 en Tabla 3). Dentro del clado Scurria, la divergencia entre las secuencias varió entre $15 \%$ y $16 \%$. En cambio, para ' $L$ '. orbignyi la divergencia en relación al resto de los taxa analizados fue de $26 \%$, siendo este el porcentaje más alto registrado.

Por otra parte, el análisis de parsimonia entregó un solo árbol (Fig. 1). De un total de 724 caracteres analizados: 467 fueron constantes, 104 fueron filogenéticamente no informativos y 153 fueron informativos. La longitud del árbol 
TABLA 3

Distancias absolutas entre las 17 muestras analizadas de patelogastrópodos intermareales. Análisis de la región 16S: 724 pares de bases secuenciadas. La nomenclatura utilizada corresponde a: (1-11) corresponden a 10 especies reconocidas como tales en la literatura Scurria ceciliana, S. boehmita, S. plana, S. scurra, S. viridula, S. zebrina, S. araucana, S. variabilis, S. parasitica con las formas que habitan sobre Fissurella crassa (S. parasitica-Fiss) y sobre Enoplochiton niger (S. parasiticaEn) y 'Lottia' orbignyi; (12-13) corresponden a dos formas únicas registradas entre los $25^{\circ} \mathrm{S}$ y $28^{\circ}$ S (SVSP1) y en Pelancura (SVSP2); (14-16) corresponden a formas que habitan sobre las valvas del mitílido Perumytilus purpuratus (CP1, CP2 y CP3); (17) corresponde a aquellos que habitan pozas intermareales en Chile central (PO)

Absolute distances between the 17 intertidal limpet samples analyzed. Region 16S: 724 base pairs sequenced. The nomenclature used is as follows: (1-11) corresponding to 10 species which are reported in the literature: Scurria ceciliana, S. boehmita, S. plana, S. scurra, S. viridula, S. zebrina, S. araucana, S. variabilis, S. parasitica on Fissurella crassa (S. parasitica-Fiss), S. parasitica on Enoplochiton niger (S. parasitica-En) and 'Lottia' orbignyi; (12-13) corresponding to two forms registered between $25^{\circ} \mathrm{S}-28^{\circ} \mathrm{S}$ (SVSP1) and in Pelancura (SVSP2); (14-16) corresponding to forms inhabiting on valves of the mussel Perumytilus purpuratus (CP1, CP2 y CP3); (17) corresponding to limpets inhabiting intertidal pools in central Chile (PO)

\begin{tabular}{|c|c|c|c|c|c|c|c|c|c|c|c|c|c|c|c|c|c|}
\hline & 1 & 2 & 3 & 4 & 5 & 6 & 7 & 8 & 9 & 10 & 11 & 12 & 13 & 14 & 15 & 16 & 17 \\
\hline 1. Scurria ceciliana & 0 & & & & & & & & & & & & & & & & \\
\hline 2. Scurria boehmita & 1 & 0 & & & & & & & & & & & & & & & \\
\hline 3. Scurria plana & 85 & 85 & 0 & & & & & & & & & & & & & & \\
\hline 4. Scurria scurra & 53 & 52 & 82 & 0 & & & & & & & & & & & & & \\
\hline 5. Scurria viridula & 108 & 107 & 110 & 108 & 0 & & & & & & & & & & & & \\
\hline 6. Scurria zebrina & 115 & 116 & 116 & 119 & 48 & 0 & & & & & & & & & & & \\
\hline 7. Scurria araucana & 58 & 57 & 85 & 18 & 107 & 119 & 0 & & & & & & & & & & \\
\hline 8. Scurria variabilis & 94 & 93 & 100 & 91 & 129 & 133 & 94 & 0 & & & & & & & & & \\
\hline 9. Scurria parasitica-Fiss & 94 & 93 & 100 & 91 & 129 & 133 & 94 & 0 & 0 & & & & & & & & \\
\hline 10. Scurria parasitica-En & 94 & 93 & 100 & 91 & 129 & 133 & 94 & 0 & 0 & 0 & & & & & & & \\
\hline 11. 'Lottia' orbignyi & 135 & 134 & 139 & 131 & 149 & 155 & 135 & 126 & 126 & 126 & 0 & & & & & & \\
\hline 12. SVSP1 & 95 & 94 & 102 & 92 & 131 & 134 & 95 & 2 & 2 & 2 & 128 & 0 & & & & & \\
\hline 13. SVSP2 & 94 & 93 & 100 & 91 & 129 & 133 & 94 & 0 & 0 & 0 & 126 & 2 & 0 & & & & \\
\hline 14. CP1 & 1 & 1 & 84 & 52 & 107 & 116 & 57 & 93 & 93 & 93 & 134 & 94 & 93 & 0 & & & \\
\hline 15. CP2 & 53 & 54 & 83 & 9 & 108 & 118 & 15 & 94 & 94 & 94 & 132 & 95 & 94 & 54 & 0 & & \\
\hline 16. $\mathrm{CP} 3$ & 48 & 48 & 85 & 53 & 107 & 120 & 51 & 96 & 96 & 96 & 130 & 98 & 96 & 47 & 55 & 0 & \\
\hline 17. $\mathrm{PO}$ & 57 & 56 & 84 & 18 & 108 & 117 & 4 & 90 & 90 & 90 & 131 & 91 & 90 & 56 & 15 & 49 & 0 \\
\hline
\end{tabular}

TABLA 4

Diferencias en las sustituciones de pares de secuencias para el gen 16S para conespecíficos del género Scurria (basadas en todos los sitios) una vez removidas las regiones ambiguas. Nomenclatura como en la Tabla 3

Substitution differences among sequence pairs for gene 16S for Scurria conspecifics (all sites) and after removing ambiguous regions. Nomenclature as in Table 3

\begin{tabular}{lcc}
\hline \multicolumn{1}{c}{ Par } & $\begin{array}{c}\text { Transiciones } \\
\text { (número* total de sitios) }\end{array}$ & $\begin{array}{c}\text { Transversiones } \\
\text { (número* total de sitios) }\end{array}$ \\
\hline S. variabilis, S. parasitica-Fiss & $0 / 643$ & $0 / 643$ \\
S. variabilis, S. parasitica-En & $0 / 659$ & $0 / 659$ \\
S. variabilis, SVSP1 & $2 / 639$ & $0 / 639$ \\
S. variabilis, SVSP2 & $0 / 656$ & $0 / 656$ \\
S. parasitica-Fiss, S. parasitica-En & $0 / 642$ & $0 / 642$ \\
S. parasitica-Fiss, SVSP1 & $2 / 622$ & $0 / 622$ \\
S. parasitica-Fiss, SVSP2 & $0 / 639$ & $0 / 639$ \\
S. parasitica-En, SVSP1 & $2 / 639$ & $0 / 639$ \\
S. parasitica-En, SVSP2 & $0 / 656$ & $0 / 656$ \\
SVSP1, SVSP2 & $2 / 639$ & $0 / 639$ \\
S. araucana, PO & $2 / 650$ & $2 / 650$ \\
S. scurra, CP2 & $9 / 630$ & $0 / 630$ \\
S. ceciliana, S. boehmita & $1 / 628$ & $0 / 628$ \\
S. ceciliana, CP1 & $1 / 626$ & $0 / 626$ \\
S. boehmita, CP1 & $1 / 651$ & $0 / 651$ \\
\hline
\end{tabular}




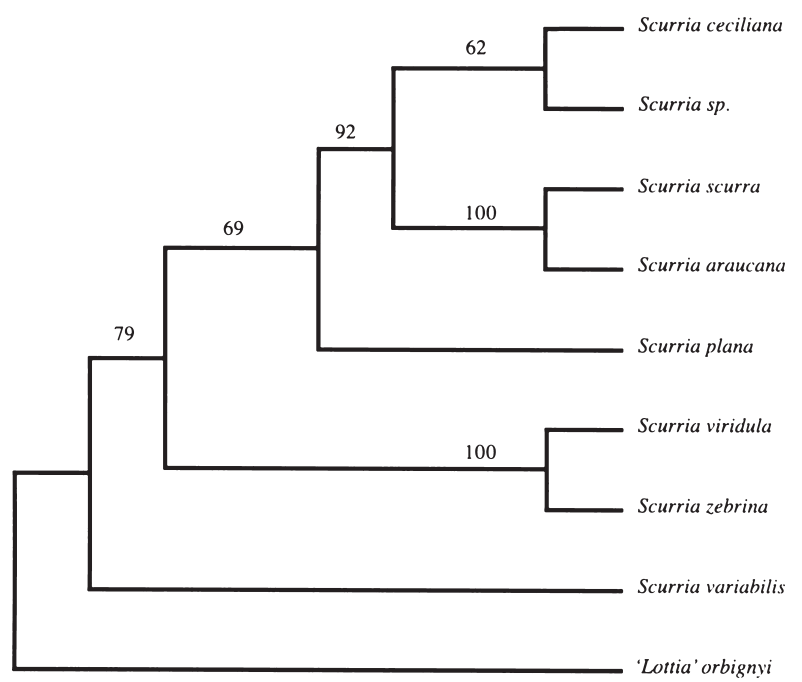

Fig. 1: Hipótesis filogenética obtenida a partir del análisis de máxima parsimonia de la región 16S de ADN mitocondrial para los patelogastrópodos intermareales endémicos de la costa rocosa de Chile-Perú. Sobre las ramas se ubican los valores de remuestreo ("bootstrap", 1.000 réplicas).

Maximum parsimony phylogenetic hypothesis for region $16 \mathrm{~S}$ of mitochondrial DNA for endemic rocky shore intertidal limpets from the coast of Chile-Perú. On branches are shown resampling values (bootstrap, 1,000 replicates).

fue de 446 pasos, con un índice de consistencia de 0,751 y un índice de retención igual a 0,587. A su vez, el análisis de máxima probabilidad también entregó como resultado un solo árbol cuya topología fue idéntica al obtenido por máxima parsimonia (Fig. 2). Tanto con máxima parsimonia como máxima probabilidad, los valores de remuestreo "bootstrap" fueron superiores a $62 \%$. Cabe mencionar que debido a que 'Lottia' orbignyi fue la especie más distante en relación al resto de los taxa analizados (Tabla 3 ), esta se utilizó como grupo externo para enraizar el árbol. De acuerdo con la hipótesis filogenética (Fig. 1 y Fig. 2), la rama basal está representada por $S$. variabilis, especie que aparece definiendo al resto de los miembros del género Scurria. Del mismo modo, se encontró un fuerte soporte al clado compuesto por las especies restantes (79 y 97 \% según análisis de parsimonia y máxima probabilidad respectivamente). La resolución del clado conformado por $S$. viridula y S. zebrina fue del $100 \%$. A su vez, $S$. plana es la especie basal a los clados de especies más derivadas conformados por $S$. scurra-S. araucana y $S$. ceciliana-Scurria sp. con una resolución que varió entre 62 y $100 \%$.

\section{Clasificación propuesta}

En la Tabla 5 se entrega un sistema de clasificación que expresa las relaciones de parentesco, obtenidas a partir del análisis filogenético de caracteres moleculares, de las especies de patelogastrópodos estudiadas (Fig. 1 y Fig. 2). Además, se entrega información respecto de: la estructura y morfología de la concha (Fig. 3), dientes de la rádula (Fig. 4), aspectos de la anatomía corporal (Fig. 5), distribución, hábitat y aspectos ecológicos de las especies estudiadas. En el texto se presentan las especies según su posición dentro de la filogenia (Fig. 1 y Fig. 2), desde la más basal hasta las especies que conforman los clados más derivados. Adicionalmente, en la Tabla 6 se entrega un resumen de los principales caracteres de la estructura de la concha y morfología corporal de las especies de patelogastrópodos estudiadas. Para cada especie se entrega información ecológica relevante.

\section{Lottiidae (Gray, 1840)}

De acuerdo con Lindberg (1988a) las características que presentan los miembros de esta familia son: concha cónica, compuesta de cuatro a seis capas incluyendo el miostraco; capa interna radial y/o lamelar cruzada compleja seguida del miostraco, en algunos casos con una capa lamelar cruzada radial, luego viene una capa lamelar cruzada concéntrica y finalmente una capa externa prismática. Ausencia de capas foliadas (Lindberg 1990, 1998). Rádula docoglosa con tres pares de dientes laterales. Con o sin dientes marginales. Estructuras respiratorias variables. Cretácico temprano hasta el presente (Lindberg 1988b). 


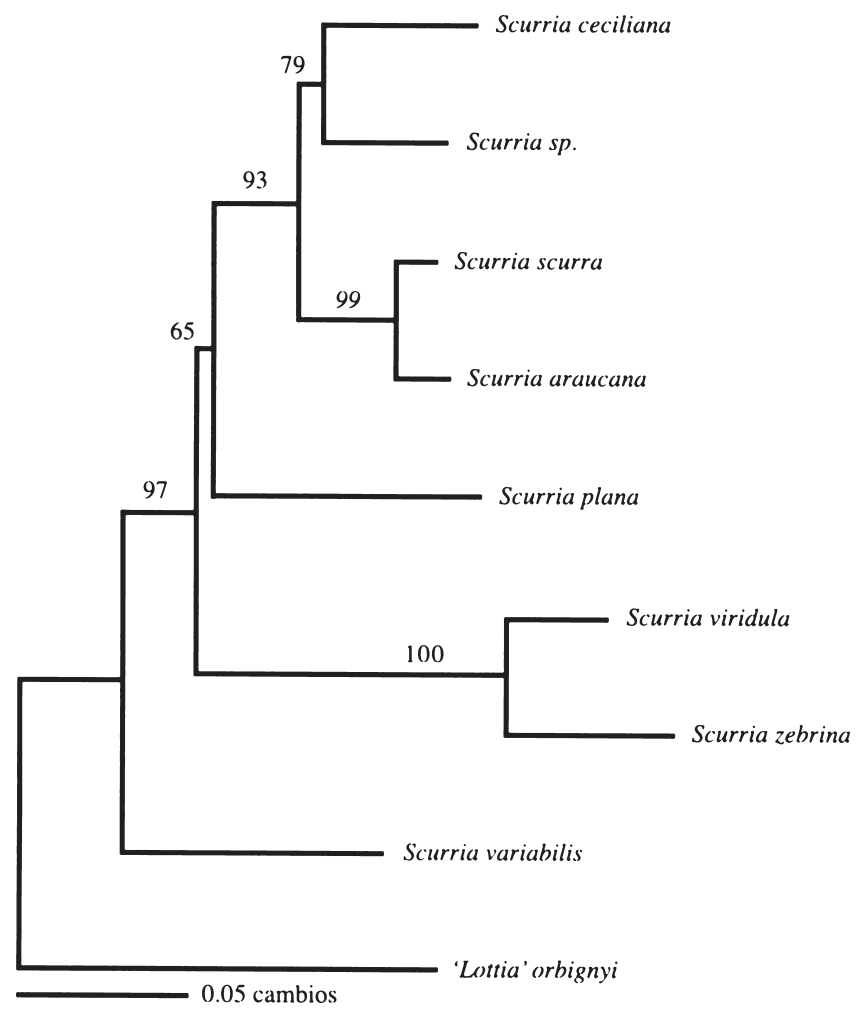

Fig. 2: Relaciones filogenéticas entre los patelogastrópodos endémicos del intermareal rocoso de Chile-Perú inferido por máxima probabilidad a partir del análisis de la región 16S de ADN mitocondrial. Sobre las ramas se ubican los valores de remuestreo ("bootstrap", 1.000 réplicas).

Phylogenetic relationships between endemic rocky shore limpets from Chile-Perú, according to maximum likelihood analysis for region $16 \mathrm{~S}$ of mitochondrial DNA. On branches are shown resampling values (bootstrap, 1,000 replicates).

\section{TABLA 5}

Clasificación propuesta en este trabajo para los patelogastrópodos intermareales de la familia Lottiidae presentes a lo largo de la costa rocosa de Chile y Perú. La clasificación se propone en función del análisis filogenético del gen mitocondrial $16 \mathrm{~S}$ y de acuerdo con el estudio comparativo de la estructura y morfología de la concha, dientes de la rádula y anatomía corporal

Proposed classification according to this study for the intertidal limpets of the family Lottidae registered along the rocky shore of Chile and Perú. The classification is based on the phylogenetic study of molecular characters (16S mitochondrial gene), and according to comparative study of shell structure, morphology, color pattern, radular teeth and corporal anatomy

\begin{tabular}{c}
\hline Clasificación propuesta en este estudio \\
\hline Familia Lottiidae (Gray, 1840) \\
Subfamilia Lottiinae (Gray, 1840) \\
Tribu Lottiini (Lindberg, 1988b) \\
Lottia orbignyi (Dall, 1909) \\
Tribu Scurriini (Lindberg, 1988b) \\
Scurria variabilis (Sowerby, 1839) \\
Scurria zebrina (Lesson, 1830) \\
Scurria viridula (Lamarck, 1819) \\
Scurria plana (Philippi, 1846) \\
Scurria araucana (Orbignyi, 1841) \\
Scurria scurra (Lesson, 1830) \\
Scurria ceciliana (Orbignyi, 1841)
\end{tabular}


(A)
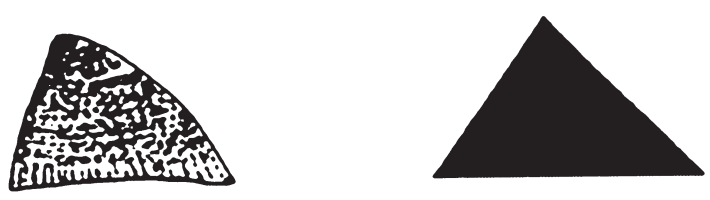

Perfil alto

(B)
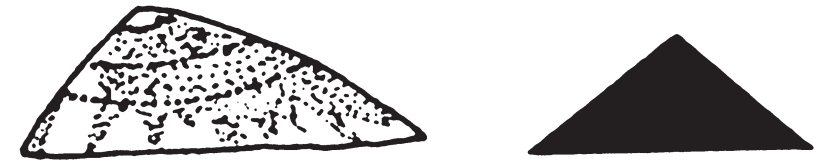

Perfil medio

(C)

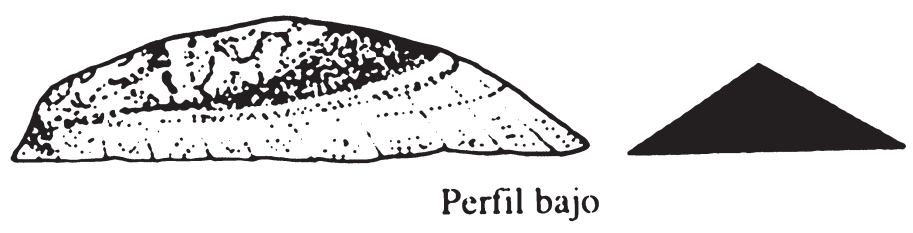

(D)

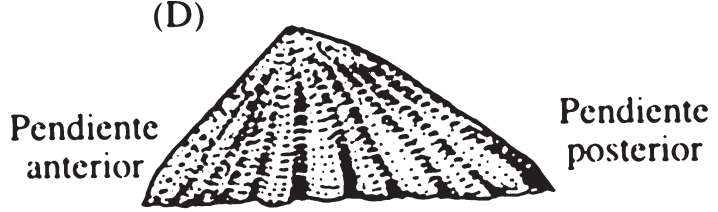

(E)

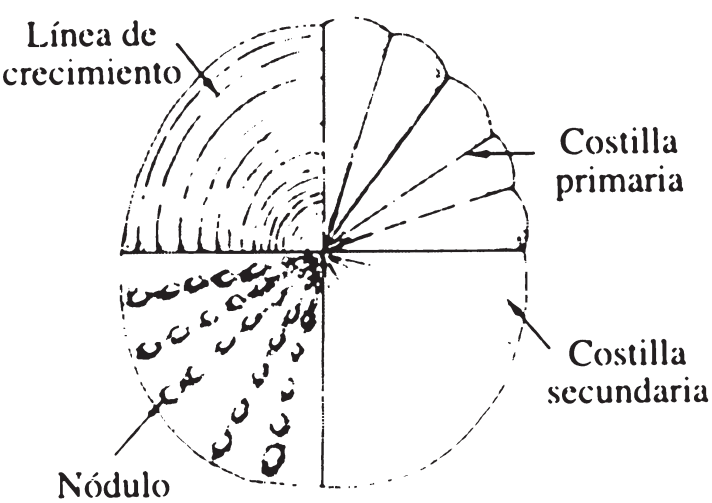

$(\mathrm{F})$

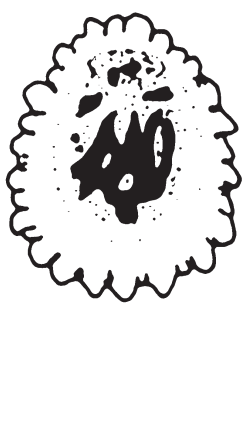

(G)

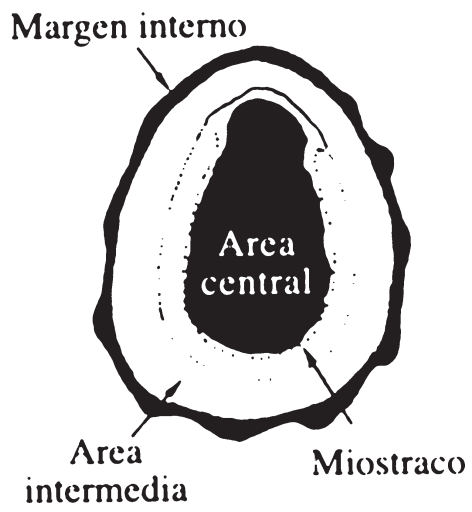

Fig. 3: Principales características relacionadas con la morfología de la concha. Morfología externa: (A-C) perfiles de la concha; (D) pendiente; (E) escultura de la concha; (F) borde de la concha crenulado. Morfología interna: (G) principales áreas que conforman el interior de la concha. Redibujado de Lindberg (1981).

Main limpet shell characteristics. External morphology: (A-C) shell profiles; (D) shell slope; (E) radial shell sculpture; (F) crenulate shell margin. Internal morphology; $(\mathrm{G})$ internally the shell is divided into four areas, which are differentiated by both color and shell texture. Redrawn from Lindberg (1981). 


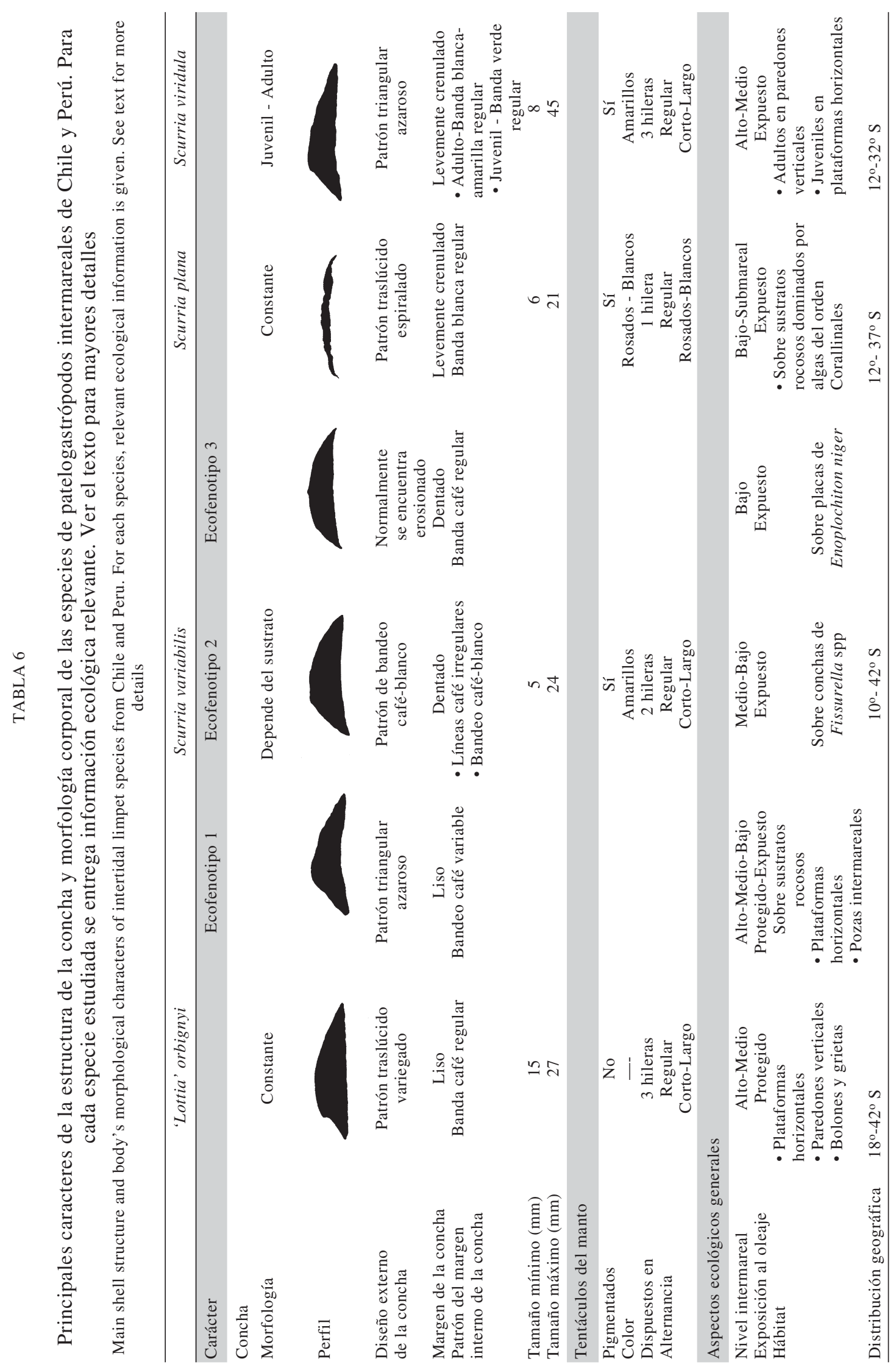




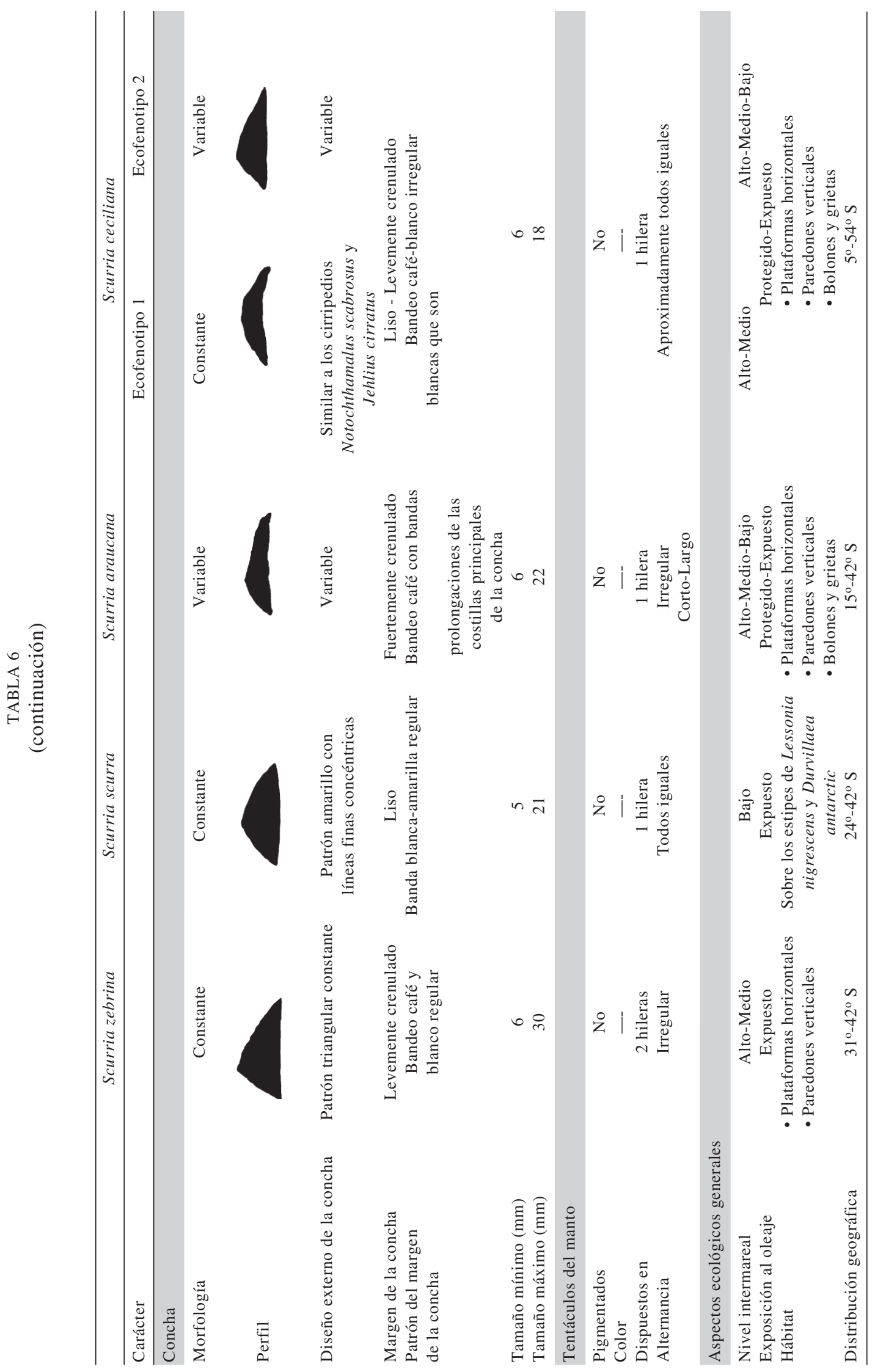




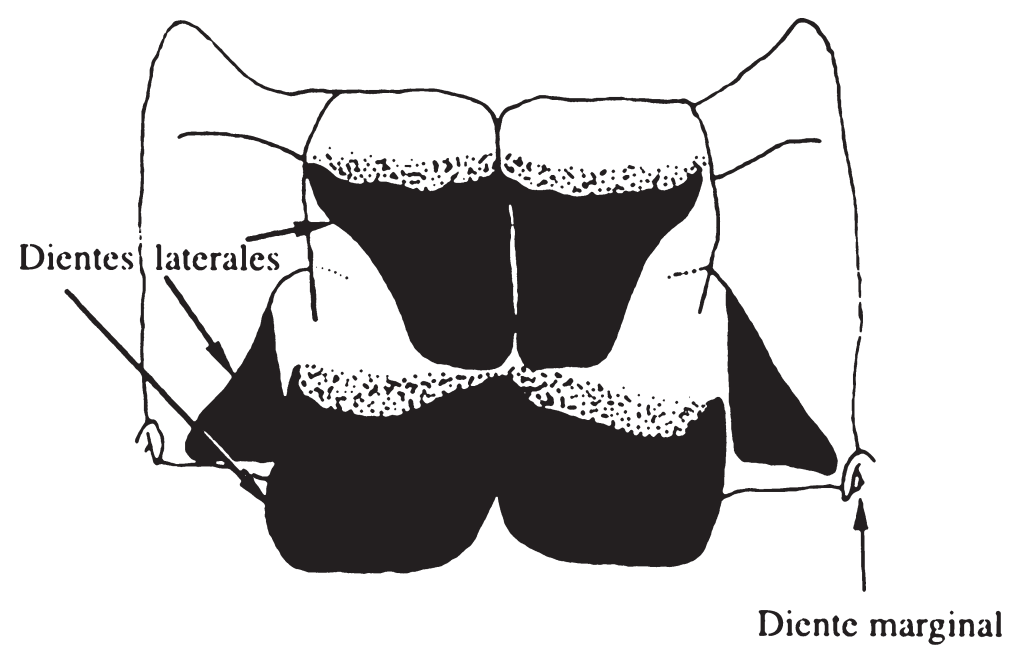

Fig. 4: Dientes que conforman la rádula en los patelogastrópodos. Redibujado de Lindberg (1981). Limpet radular teeth. Redrawn from Lindberg (1981).

(A)
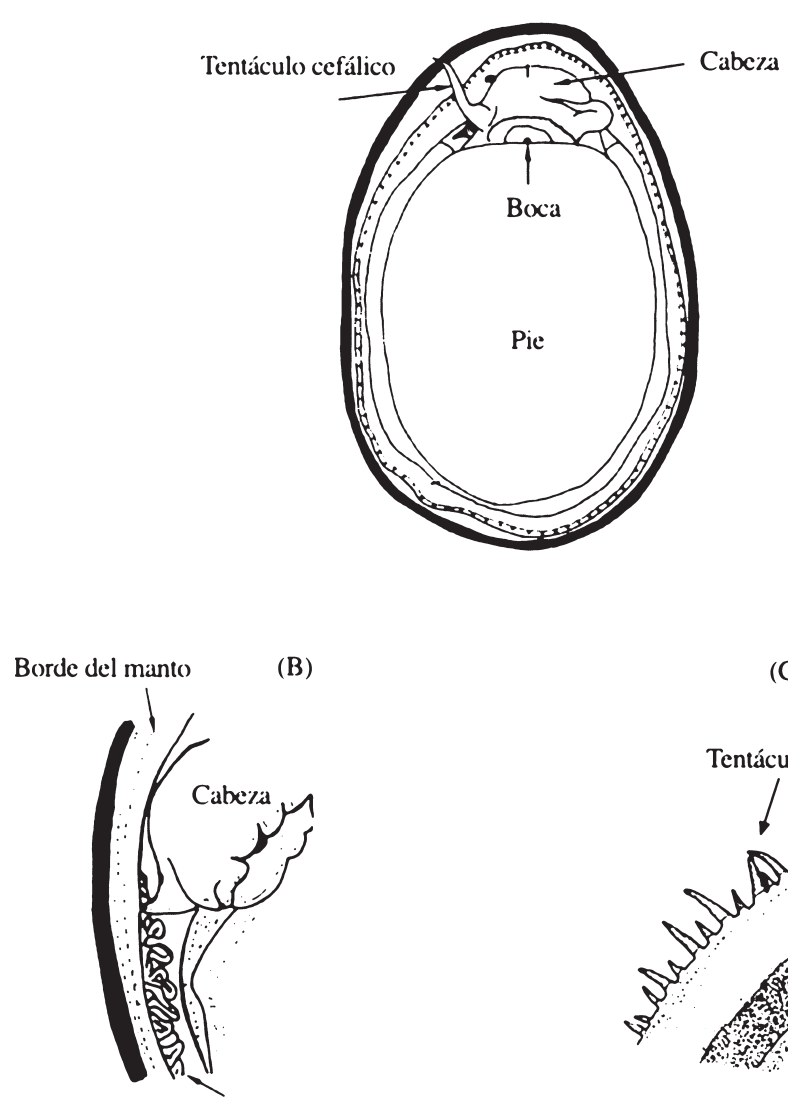

(C)

Branquia secundaria

Fig. 5: Principales aspectos de la anatomía corporal considerados para la caracterización de las especies de patelogastrópodos estudiados. (A) vista ventral del cuerpo de un patelogastrópodo; (B) zona branquial; (C) tentáculos del manto. Redibujado de Lindberg (1981).

Main aspects of limpet body considered for the characterization species studied. (A) ventral view of limpet body; (B) branchial zone; (C) mantle tentacles. Redrawn from Lindberg (1981). 


\section{Lottiinae (Gray, 1840)}

Estructura de la concha con una capa externa prismática, la que a su vez está compuesta de dos capas: la interior fibrosa y la externa prismática (compleja o simple).

\section{Lottiini (Lindberg, 1988a)}

Capa prismática predominantemente fibrosa (ca. $60 \%$, ver Lindberg 1988a), seguida de una capa fina externa de estructura prismática simple. Rádula compuesta por tres pares de dientes laterales; los bordes del primer par son convexos, mientras que los del segundo par son redondeados. Detrás y sobre el tercer par de dientes hay una placa pequeña, oval y ventral. Presencia o ausencia de un par de dientes marginales. Tentáculos del manto de forma variable, así como también los plegamientos del estómago. Se distribuye a lo largo del Pacífico este, desde Alaska hasta Chile. Esta tribu formaría un clado compuesto por los géneros Lottia y Tectura (Lindberg 1990), ambos presentes en el Pacífico este (Lindberg 1988a). Los miembros de esta tribu predominan en las provincias de moluscos Aleutiana, Oregoniana, Californiana y Panameña (Lindberg 1988a), y también están presentes en la provincia Peruana, desde los $12^{\circ} \mathrm{S}$ hasta los $42^{\circ} \mathrm{S}$.

'Lottia' (Sowerby, 1834)

Estructura de la concha con capa externa del tipo prismática simple (MacClintock 1967). Ápice típicamente subcentral; escultura con líneas de crecimiento concéntricas; costillas principales radiales, con nódulos o una combinación de estas; costillas secundarias radiales. Patrón externo de color típicamente variegado; margen interior de la concha y área central usualmente coloreada, área intermedia comúnmente sin color. Rádula con tres pares laterales de dientes, generalmente desiguales en tamaño y forma; un solo par de dientes marginales reducidos o uncini, presentes en la parte posterior final de cada fila radular. Morfología y arreglo de las placas laterales compleja. En algunas especies partes del pie y la cabeza presentan pigmentación. Estructuras respiratorias variables; branquia secundaria puede o no estar presente. Bordes orales habitualmente ausentes.

'Lottia' orbignyi (Dall, 1909) (Fig. 6A)

Sinonimia: Acmaea orbignyi (Dall, 1909: 179); Collisella orbignyi (Marincovich, 1973: 20).

Concha: comparativamente grande, baja y delgada con una escultura de costillas secundarias muy delgadas. El patrón de color externo es oscuro, variando entre gris, azul y café. Interior blanco a azul con marcas irregulares café en el área central. Patrón translúcido variegado. Margen de la concha oscuro y claramente demarcado. Tamaño: 15-27 mm.

Anatomía: los tentáculos del manto se alternan entre corto y largo y están arreglados en un sistema de tres hileras, al igual que en Scurria viridula. Cordón branquial ausente.

Distribución: San Pablo, Perú (18 $\left.{ }^{\circ} \mathrm{S}\right)$ hasta Pirulil, Isla de Chiloé, Chile $\left(42^{\circ} \mathrm{S}\right)$.

Ecología: intermareal rocoso, presente en paredones verticales y bolones de tamaño variable. En áreas expuestas y/o protegidas del oleaje. Al parecer, esta especie es sensible a la desecación por lo que generalmente se encuentra en áreas que no están sujetas a la exposición directa del sol. También permanece en grietas durante el día. Ante el contacto directo o indirecto con el asteroideo Heliaster helianthus (Lamarck, 1816), la especie responde con: elevación de la concha, torsión del pie y movimientos de la concha de un lado para otro (Espoz \& Castilla 2000). De acuerdo con la información entregada por Espoz \& Castilla (2000), a esta reacción le sigue una respuesta activa de tipo locomotora, la cual ocurre dentro de los primeros cinco segundos desde la ocurrencia del primer contacto. Llama la atención el que esta respuesta de escape frente al depredador muestre una variación geográfica, correlacionada con un gradiente en las densidades del depredador $H$. helianthus. La respuesta se hace latitudinalmente menos vigorosa a medida que la densidad del asteroideo decae hacia el extremo sur de Chile (C. Espoz observaciones personales).

Observaciones: el patrón translúcido variegado es similar al registrado en Tectura persona (Rathke, 1833) en el Pacífico noreste.

\section{Scurriini (Lindberg, 1988a)}

La capa fibrosa que forma parte de la capa externa prismática es más delgada que en Lottiini $(<20 \%$ de la capa prismática) (Lindberg 1988a). Como otros miembros de la subfamilia Lottiinae, en la tribu Scurriini se registra un rango marcado de variación en la morfología de la concha. La rádula está compuesta por tres pares de dientes laterales; los bordes medios del primer par son típicamente convexos, el segundo par posee un borde medio redondeado. Detrás del tercer par de dientes laterales se observa una placa ventral pequeña y oval. Con o sin dientes marginales. Tentáculos 
(A)
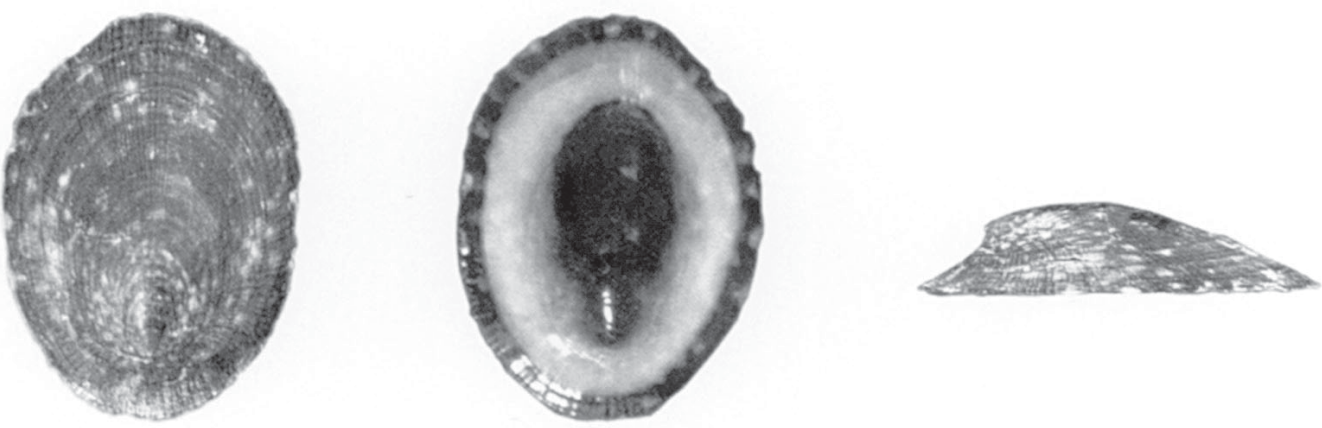

(B)
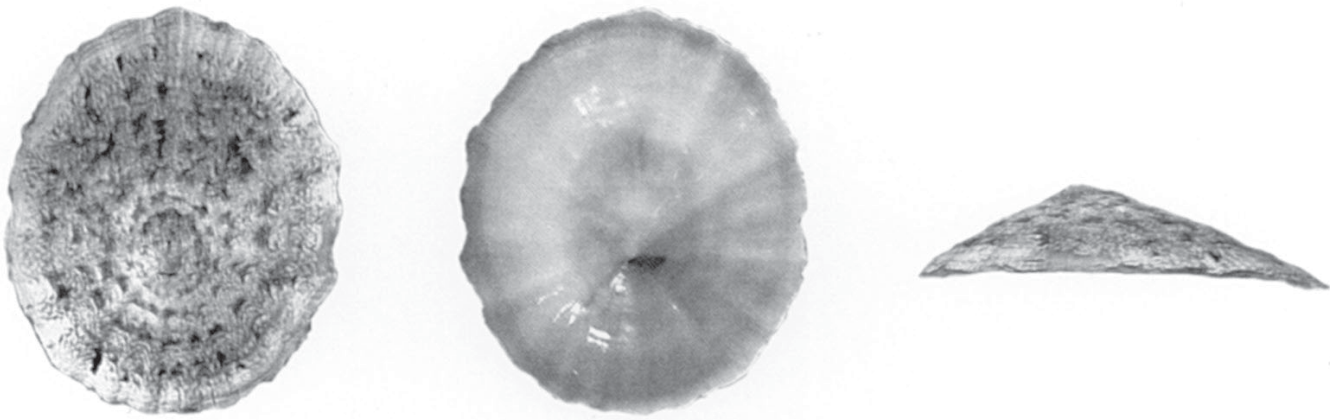

(C)
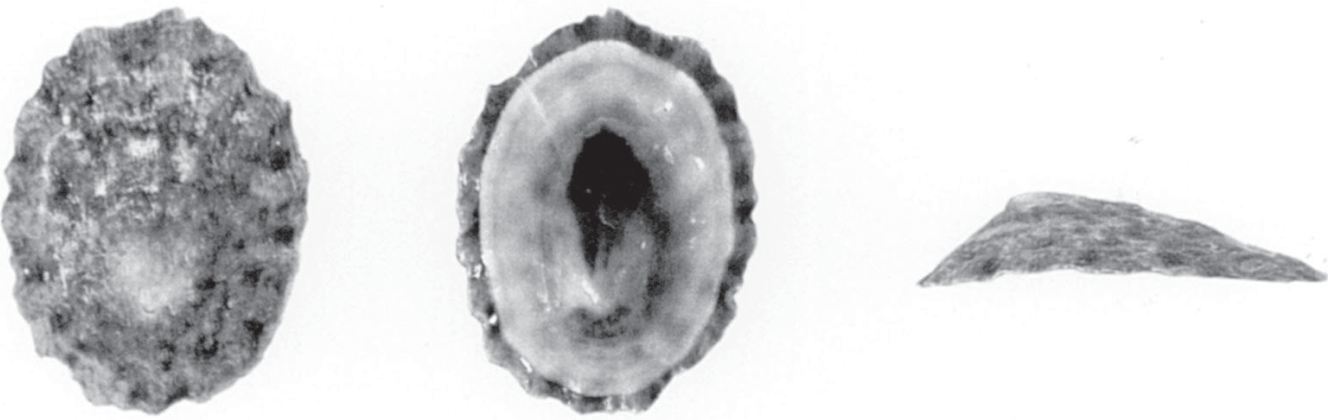

(D)
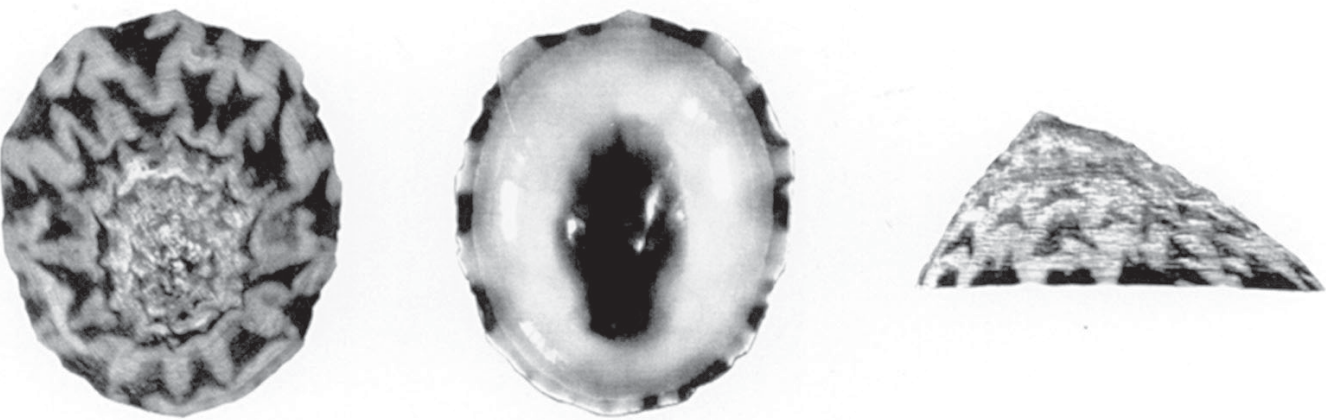

Fig. 6: Morfología de la concha. (A) 'Lottia' orbignyi, longitud total 21,45 mm; (B) ejemplar adulto de Scurria viridula, longitud total $34,05 \mathrm{~mm}$; (C) juvenil de S. viridula, longitud total 12,07 $\mathrm{mm}$; (D) S. zebrina, longitud total $18,50 \mathrm{~mm}$.

Shell morphology. (A) 'Lottia' orbignyi, total length $21.45 \mathrm{~mm}$; (B) Scurria viridula, (adult), total length $34.05 \mathrm{~mm}$; (C) S. viridula (juvenile) total length $12.07 \mathrm{~mm}$; (D) S. zebrina, total length $18.50 \mathrm{~mm}$. 
del manto y plegamientos del intestino de forma variable. Dos géneros, presentes en el $\mathrm{Pa}-$ cífico este, son referidos a esta tribu, Scurria en el hemisferio sur y Discurria en el hemisferio norte (Lindberg 1988a, 1990).

\section{Scurria (Gray, 1847)}

Estructura de la concha con capa externa del tipo prismática compleja (MacClintock 1967). La rádula consiste en tres pares de dientes laterales, de los cuales el segundo es el de mayor tamaño, salvo en el caso de $S$. plana. El primer par de dientes es típicamente convexo hacia el interior y con bordes externos cóncavos. La placa basal del tercer par de dientes lateral es pequeña, oval y ventral. En algunas especies, como en el caso de S. scurra, esta estructura está mineralizada y es blanca opaca antes que translúcida. Se observa la presencia de un par de dientes marginales o uncini. Tentáculos del manto de tamaño y posición variable, al igual que el patrón de plegamiento del estómago. Presencia de un cordón branquial en todas las especies, salvo en $S$. araucana y $S$. ceciliana. El género se distribuye desde Máncora, Perú ( $\left.4^{\circ} \mathrm{S}\right)$ hasta Isla Navarino, Chile $\left(56^{\circ} \mathrm{S}\right)$.

A continuación se describe cada una de las especies pertenecientes a este clado, desde la especie más basal hasta las especies que conforman el clado más derivado (ver Fig. 1 y Fig. 2).

\section{Scurria variabilis (Sowerby, 1839) (Fig. 7)}

Sinonimia: Lottia variabilis (Sowerby, 1839:147); Patella parasitica (Orbigny, 1841:481); Acmaea nisoria (Philippi, 1846: 49); Lottia cymbiola (Gould, 1846:151); Acmaea leucophaea (Philippi, 1846: 22); Patella coffea (Reeve, 1855; vol. 8, pl. 41, figuras 139 a y b); Patella variabilis (Reeve, 1855); Patella penicillata (Reeve, 1855: 34, pl. 102a, b); Acmaea variabilis (Pilsbry, 1891; vol. 13: 3435; Dall, 1909: 237; Thiem, 1917: 617); Scurria parasitica (Pilsbry, 1891: 63; Dall, 1909; Marincovich, 1973: 21; Alamo \& Valdivieso, 1987: 16); Acmaea coffea (Dall, 1909: 237; Thiem, 1917: 614); Acmaea parasitica (Thiem, 1917: 616); Collisella variabilis (Marincovich, 1973: 20); Collisella huppeana (Ramírez, 1974: 24); Collisella philippiana (Ramírez, 1974: 27); Collisella silvana (Ramírez, 1974: 29).

Concha: concha oval. En esta especie tanto el patrón externo como el interno de la concha varía dependiendo del sustrato en el cual habi- ten los especímenes. Los ejemplares que habitan sobre sustratos rocosos cubiertos por algas coralinas (Fig. 7A) son de concha blanca y moteada con marcas oscuras, gris y café. El color blanco de la concha es el producto de manchas blancas reticuladas separadas por canales de material translúcido de la concha. Internamente presentan un área oscura central caracterizada por la presencia de líneas irregulares, seguida por la marca de la inserción del músculo. El área adyacente aparece con manchas de color café y amarillo. El margen interno de la concha presenta líneas café de grosor variable, las cuales se observan tanto en juveniles como en adultos. El patrón externo de color se pierde cuando los individuos presentan conchas erosionadas (Fig. 7B). En este caso, la erosión afecta principalmente el área apical y central externa de la concha. Los ejemplares que habitan sobre las conchas de lapas del género Fissurella (Fig. 7C) presentan una concha de altura moderada, esculpida con costillas secundarias finas. Externamente la concha presenta una apariencia estriada con líneas suaves blancas y café. Internamente, el borde de la concha presenta líneas café; el área central tiene marcas irregulares café, la marca del músculo es gris y el área entre la marca y el margen varía desde azul a café. Un rasgo característico de los ejemplares que habitan sobre las placas de Enoplochiton niger (Barnes, 1824) (Fig. 7D) es el color interno oscuro de la concha, generalmente café. Además, en estos casos las conchas aparecen externamente muy erosionadas, debido a lo cual la región del ápice se hace indistinguible. Una característica resaltante de esta especie es que el borde de la concha no es liso y presenta un patrón dentado, el cual se hace perceptible al tacto. Tamaño: 5-24 mm.

Anatomía: tentáculos del manto de color amarillo alternados en una secuencia de uno largo seguido por uno corto, arreglados en dos hileras. La franja oral está compuesta por dos capas que conforman un margen intrincado. Cordón branquial presente.

Distribución: desde Tuquillo, Perú $\left(10^{\circ} \mathrm{S}\right)$ hasta Chepu, Isla de Chiloé, Chile $\left(42^{\circ} \mathrm{S}\right)$.

Ecología: intermareal rocoso medio a inferior. En el nivel intermareal inferior, la especie está comúnmente asociada con algas coralinas incrustantes. En Chile centro y sur se encuentra también sobre las conchas de las lapas Fissurella crassa (Lamarck, 1822) y F. limbata (Sowerby, 1835). En el norte de Chile y sur del Perú, habita sobre las conchas de $S$. viridula y sobre las placas de Enoplochiton niger. Ocasionalmente, se le encuentra sobre las superficies de Chiton granosus (Frembly, 
(A)

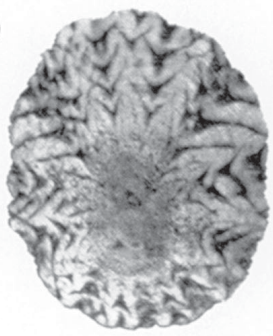

(B)

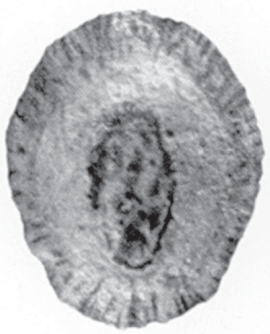

(C)

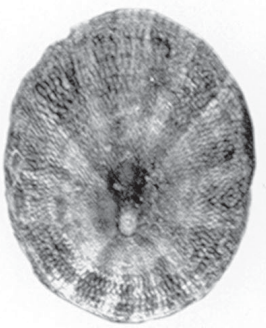

(D)

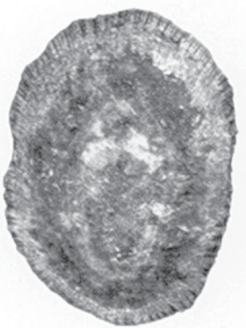

(E)

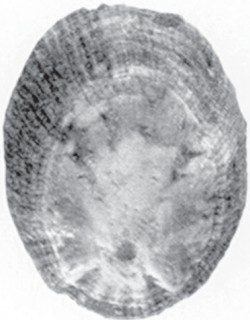

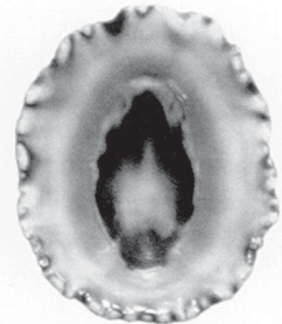
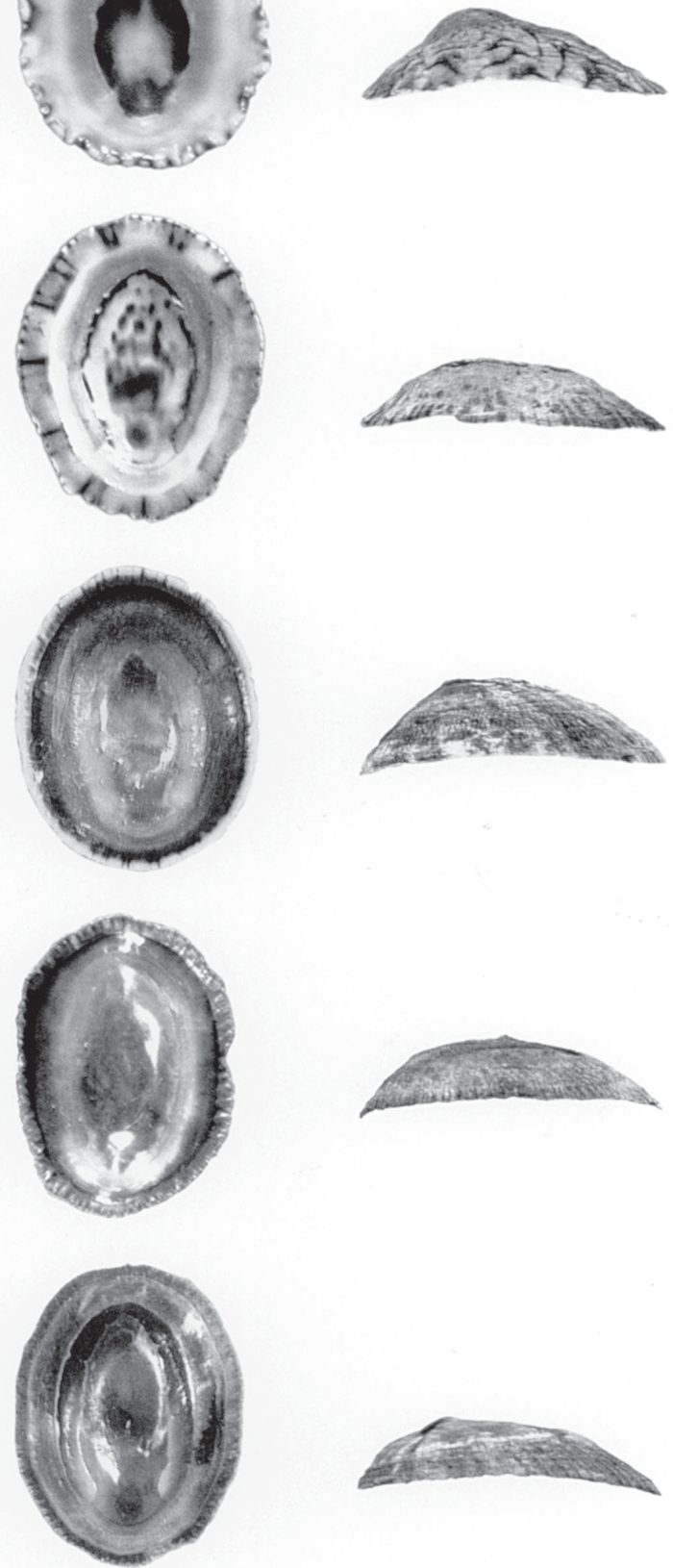

Fig. 7: Morfología de la concha de Scurria variabilis. Ejemplares que habitan sobre: (A) sustrato rocoso, longitud total 13,25 $\mathrm{mm}$; (B) sustrato rocoso con conchas erosionadas, longitud total 27,95 $\mathrm{mm}$; (C) conchas de lapas del género Fissurella, longitud total $15,15 \mathrm{~mm}$; (D) placas del chitón Enoplochiton niger, longitud total $15,50 \mathrm{~mm}$; (E) forma transicional registrada entre $10 \mathrm{~s} 25^{\circ} \mathrm{S}$ y $28^{\circ}$ $\mathrm{S}$, longitud total $15,40 \mathrm{~mm}$.

Scurria variabilis shell morphology. (A) Individuals inhabiting rocky substrata, total length $13.25 \mathrm{~mm}$; (B) individuals with eroded shell, living on rocky substrata, total length $27.95 \mathrm{~mm}$. Individuals living on: (C) shells of key-hole limpets genus Fissurella, total length $15.15 \mathrm{~mm}$, and (D) plates of the chiton Enoplochiton niger, total length $15.50 \mathrm{~mm}$. (E) Transitional morph registered between $25^{\circ} \mathrm{S}$ and $28^{\circ} \mathrm{S}$, total length $15.40 \mathrm{~mm}$. 
1827), Tegula atra (Lesson, 1830), Concholepas concholepas (Bruguière, 1789), Nucella crassilabrum (Lamarck, 1789), Nacella clypeater (Lesson, 1831) y las valvas del mitílido Perumytilus purpuratus (Lamarck, 1819). En los casos de ejemplares que viven sobre las superficies de otros moluscos, su presencia deja una marca en las conchas o placas de los ejemplares donde se encuentra. Cuando se alimenta abandona la depresión para regresar a ella al término de su actividad trófica. La alimentación ocurre por ramoneo de algas presentes en las conchas o placas donde habita la especie. Raramente abandona la superficie en la cual habita. En el terreno se registró que los períodos de actividad se inician con el incremento del oleaje; a medida que el agua las alcanza comienzan a moverse y alimentarse. La especie presenta hábitos nocturnos, además de los diurnos.

Observaciones: el rasgo más conspicuo de la concha es un patrón de marcas en la forma de cuatro líneas blancas cada una de las cuales representa una costilla. Estas líneas van desde el ápice hacia el borde de la concha en las posiciones $1,4,8$ y 11 de un reloj. Cerca del ápice, estas costillas frecuentemente son de color café oscuro. La posición de estas costillas da a la superficie dorsal de la concha una apariencia angular. Este carácter distintivo está presente principalmente en juveniles, pudiendo desaparecer en estados más avanzados de crecimiento. Las costillas restantes son amplias y bajas. Este carácter aparece compartido con numerosas especies asociadas a sustratos de carbonato de calcio que habitan hacia el norte en Panamá tropical y el Caribe (D.R. Lindberg, observaciones personales).

\section{Scurria plana (Philippi, 1846) (Fig. 8A)}

Sinonimia: Acmaea plana (Philippi, 1846: 22; Reeve, 1855, pl. 40, fig. 133; Pilsbry, 1891: 3335 [como S. viridula y S. araucana]; Dall, 1909: 280 [como S. viridula]).

Concha: la concha muestra un crecimiento en espiral en lugar de un crecimiento a través de la adición de capas en el plano anterior/posterior. Esta característica es observable a trasluz. Perfil muy bajo, de ahí se deriva el nombre específico. El patrón de coloración externo es rosado debido al sobre crecimiento de algas coralinas incrustantes pertenecientes al orden Corallinales. Tamaño: 6-21 mm.

Anatomía: los tentáculos del manto son pequeños y se presentan en un número mayor que el resto de los miembros de este clado. A su vez, los tentáculos están ordenados en una fran- ja con un patrón de alternancia entre grupos de tentáculos rosados y blancos.

Distribución: desde Naplo, Perú $\left(12^{\circ} \mathrm{S}\right)$ hasta Lebu, Chile $\left(37^{\circ} \mathrm{S}\right)$.

Ecología: habita en el intermareal rocoso inferior en superficies cubiertas por algas incrustantes pertenecientes al orden Corallinales. El sobrecrecimiento de estas algas sumado a lo bajo del perfil de la concha le da a la especie una apariencia críptica por lo que es difícil su detección en el terreno. La especie fue redescubierta por los autores en El Quisco, Chile central, luego de 147 años desde que fue originalmente descrita por Philippi en el año 1846 como Acmaea plana (Colección de patelogastrópodos del Museo Nacional de Historia Natural, Chile). La especie también habita en el submareal somero. Ocasionalmente, se registra sobre las conchas de Tegula atra que están cubiertas por coralinas incrustantes (C. Espoz observaciones personales). La formación de la depresión donde habita $S$. plana parece ser mediada por una disolución química (i.e., no es excavada con la rádula). Existen al menos dos ecofenotipos: uno asociado con el alga Gelidium chilense (Montagne) en Chile central $\left(33^{\circ} \mathrm{S}\right)$ y un segundo que habita sobre la fase crustosa de un alga Scytosiphonal en el norte de Chile $\left(25^{\circ} \mathrm{S}\right)$. En el laboratorio, la especie presenta hábitos nocturnos. Mientras se alimenta, el contacto con el asteroideo Heliaster helianthus hace que $S$. plana vuelva inmediatamente a su depresión. Al hacerlo, primero ubica el pie sobre la depresión y luego gira el cuerpo hasta que la concha se ajusta completamente a la marca de la depresión (C. Espoz observaciones personales). Este comportamiento aparece como único entre los patelogastrópodos del mundo (D.R. Lindberg, observaciones personales).

Scurria viridula (Lamarck, 1819) (Fig. 6B y 6C)

Sinonimia: Patella viridula (Lamarck, 1819: 334); Acmaea viridula (Pilsbry, 1891: 32; Dall, 1909: 178; Dell, 1971: 199); Scurria viridula (Thiem, 1917: 614; Marincovich, 1973: 23; Alamo y Valdivieso, 1987: 16).

Concha: en juveniles, la concha es delgada y frágil. Presenta un patrón externo de bandeo en secuencia entre una banda de color verde intenso seguido por bandas de color gris y verde menos intenso. Las bandas se proyectan hacia el margen de la concha por la superposición de pequeños triángulos. El margen de la concha está compuesto por una capa más delgada de color verde y claramente 
(A)
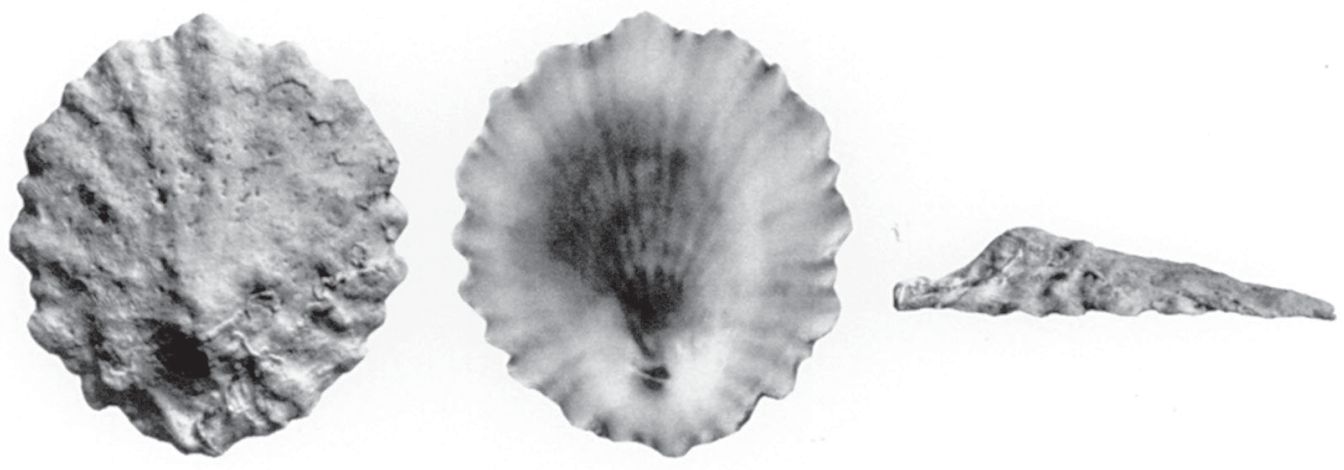

(B)
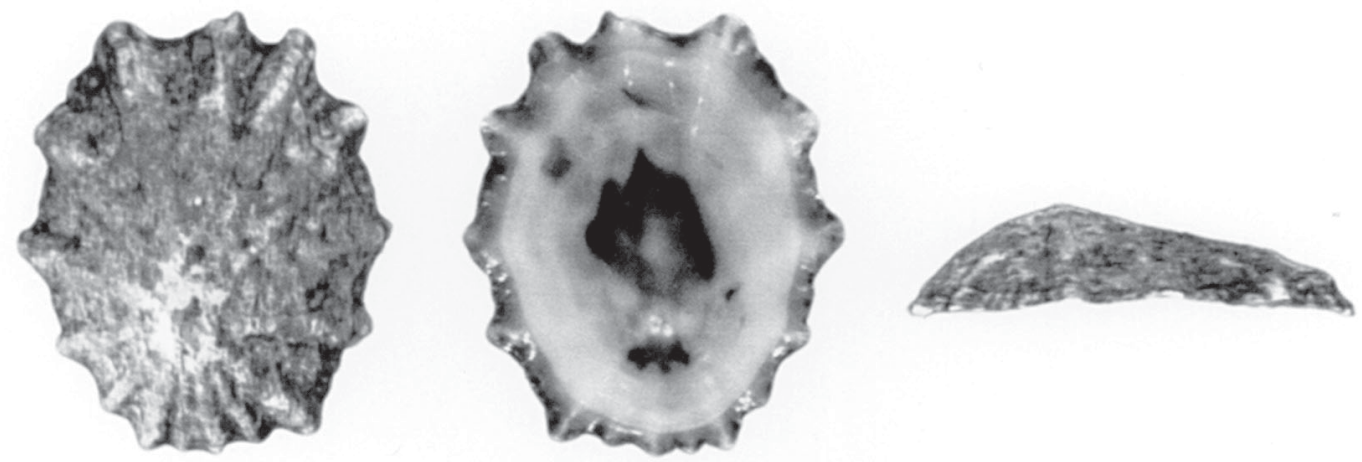

(C)
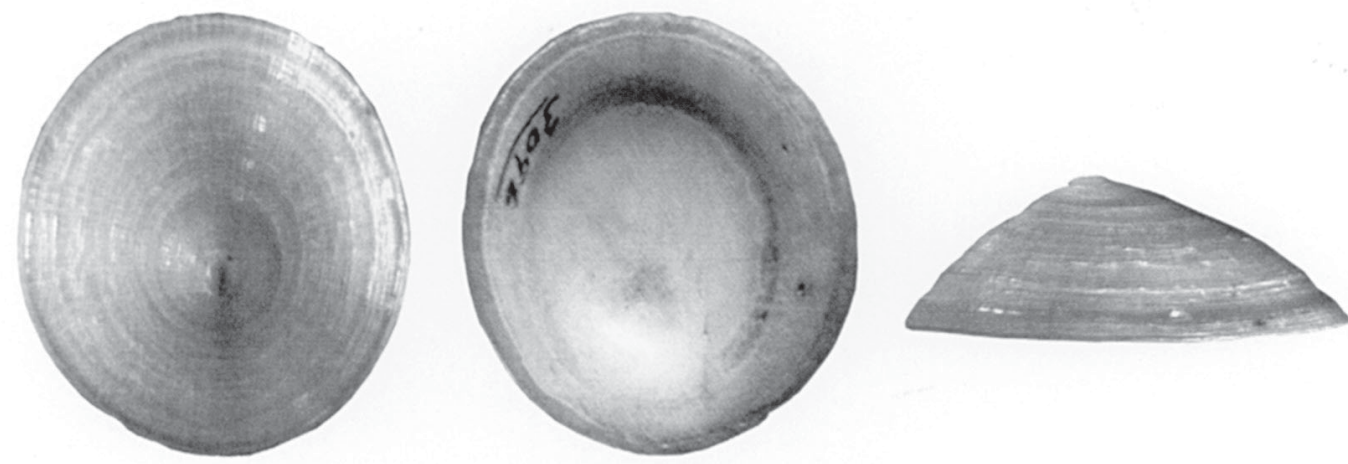

Fig. 8: Morfología de la concha. (A) Scurria plana, longitud total 19,22 mm; (B) S. araucana, longitud total 18,65 mm; (C) S. scurra, longitud total 21,35 mm.

Shell morphology. (A) Scurria plana, total length $19.22 \mathrm{~mm}$; (B) S. araucana, total length $18.65 \mathrm{~mm}$; (C) S. scurra, total length $21.35 \mathrm{~mm}$. 
diferenciada del resto de las capas de la estructura de la concha. En adultos, la concha es gruesa y fuerte, de elevación media a alta; las costillas radiales son robustas y no se prolongan más allá del margen de la concha. El patrón externo de coloración, cuando está presente, es de bandas claramente definidas de color verde, las que están formadas por pequeños triángulos superpuestos desde el ápice hacia el margen de la concha. Estas bandas se entremezclan con bandas formadas por costillas secundarias blanquecinas. Internamente, la concha es de coloración blanca a gris. En muchos casos las conchas aparecen decoloradas probablemente debido a la fuerte exposición solar a la que están sometidas en las regiones desérticas del norte de Chile y sur del Perú. Tanto en juveniles como en adultos se observan líneas concéntricas radiales. Tamaño: 8-45 mm.

Anatomía: tentáculos del manto de color amarillo arreglados en tres hileras. Son de tamaño variable alternándose entre largo y corto. Cordón branquial presente.

Distribución: desde Naplo, Perú ( $\left.12^{\circ} \mathrm{S}\right)$ hasta Zapallar-Papudo, Chile $\left(32^{\circ} \mathrm{S}\right)$.

Ecología: ocurre en sustratos rocosos desde el nivel intermareal superior al inferior, en áreas expuestas y protegidas al oleaje. Los juveniles de $S$. viridula habitan preferentemente sobre plataformas de pendiente moderada $\left(<45^{\circ}\right)$, en cambio los adultos típicamente se encuentran en paredones verticales $\left(>45^{\circ}\right)$. Ante la presencia del asteroideo Heliaster helianthus, S. viridula presenta una respuesta de escape activa de tipo locomotor similar a la registrada en 'Lottia' orbignyi (Espoz \& Castilla 2000). En el norte de Chile y sur del Perú es frecuente observar a ejemplares de $S$. variabilis sobre las conchas de S. viridula.

Observaciones: la descripción de triángulos en el patrón externo de coloración de la concha en $S$. zebrina y juveniles de $S$. viridula probablemente ha llevado a confusión, sobre todo para el material de la costa de Perú. Las conchas de ambas especies se diferencian por su robustez: S. viridula presenta conchas comparativamente más delgadas y frágiles, mientras que las conchas de $S$. zebrina son más gruesas y fuertes. Otro rasgo característico, que diferencia a los juveniles de ambas especies, es la existencia en $S$. viridula de una capa de la concha delgada y de color verde ubicada en el margen de la concha, visiblemente diferente al resto de las capas. En S. zebrina esta capa es de color café y su grosor no presenta diferencias con el resto de las capas.
Scurria zebrina (Lesson, 1830) (Fig. 6D)

Sinonimia: Patella zebrina (Lesson, 1830: 417; Orbigny 1841: 480); Scurria zebrina (Pilsbry, 1891: 62; Dall, 1909: 235; Thiem, 1917: 613); Patelloidea zebrina (Dell, 1971: 200); Collisella zebrina (Marincovich, 1973: 21; Álamo y Valdivieso, 1987: 15); Collisella aconcaguina (Ramírez, 1974: 19).

Concha: la morfología de la concha es una de las menos variables entre las especies del género Scurria. Concha grande, gruesa, cónica y de elevación alta. Patrón externo de coloración café amarillento. Costillas suavemente coloreadas con marcas triangulares oscuras, café a negro; claramente distinguibles unas de otras. Las costillas están ordenadas en bandas que se proyectan desde el ápice hacia el margen de la concha. Internamente son de color blanco amarillo, con un margen que presenta un bandeo café y blanco. Cuando los individuos son de gran tamaño presentan conchas erosionadas. Tamaño: 6-30 mm.

Anatomía: tentáculos del manto de tamaño variable ordenados en dos hileras. Cordón branquial presente.

Distribución: desde Caleta Derrumbe, Chile $\left(31^{\circ} \mathrm{S}\right)$ hasta Chepu, Isla de Chiloé, Chile $\left(42^{\circ}\right.$ $\mathrm{S})$, pero es posible que se distribuya más al sur de los $42^{\circ} \mathrm{S}$.

Ecología: Presente en el intermareal rocoso superior a medio en ambientes expuestos al oleaje. Preferentemente habita en paredones verticales donde alcanza los mayores tamaños. La presencia de esta especie en plataformas de inclinación moderada $\left(<45^{\circ}\right)$ se restringe a juveniles o adultos preferentemente de la zona sur de Chile. Ocasionalmente, ocurre en depresiones del sustrato rocoso. En estos casos, cuando se encuentra fuera de las depresiones y se enfrenta a la presencia de Heliaster helianthus, la especie muestra una respuesta de escape que consiste en evitar el contacto a través del movimiento lento en dirección a la depresión, en donde se adhiere fuertemente al sustrato (Espoz \& Castilla 2000). De acuerdo con estos autores, cuando los individuos no habitan en depresiones, el encuentro con el asteroideo es evitado a través del aumento en la fuerza de adhesión a la roca.

Observaciones: uno de los rasgos característicos de esta especie es la presencia de triángulos en la concha. Probablemente debido a la descripción de estos triángulos, juveniles de $S$. viridula pueden ser confundidos con $S$. zebrina, particularmente en el litoral de Perú, donde esta última especie está ausente. Entre los patelogastrópodos interma- 
reales endémicos de Chile y Perú, S. zebrina es la especie que presenta el rango geográfico más restringido, con $1.221 \mathrm{~km}$ lineales (Espoz 2002).

\section{Scurria scurra (Lesson, 1830) (Fig. 8C)}

Sinonimia: Patella scurra (Lesson, 1830: 421; Orbigny, 1841: 478); Acmaea cymbula (Hupé, 1854: 252); Scurria scurra (Dall, 1909: 237; Thiem, 1917: 615; Dell, 1971: 201; Marincovich, 1973: 22; Alamo y Valdivieso, 1987: 16).

Concha: perfil cónico y alto. Los ejemplares que habitan sobre las feófitas Lessonia nigrescens (Bory, 1826) y Durvillaea antarctica (Chamisso) (Hariot, 1892) presentan una concha gruesa, amarilla y con margen relativamente redondo. Costillas secundarias muy finas y concéntricas. Apice subcentral (i.e., ubicado cerca del centro). En juveniles, el ápice externamente es café. Internamente, la concha es blanquecina con margen angosto y bien definido. La especie presenta una forma transicional detectada a partir del análisis molecular (CP2 en Tabla 3). En esta forma la concha posee un perfil alto y cónico con ápice en el cuarto anterior de la concha, dirigido anteriormente. Asimismo, la apertura es irregular probablemente debido a que se encuentra sobre mitílidos bivalvos. La concha presenta una pendiente anterior recta y pendientes posterior y lateral convexas. Concha oscura café y negra con rayas blancas que corresponden a las costillas. A su vez, la concha presenta rayas delgadas blancas con manchas en los espacios intercostales. Las marcas en la región anterior de la concha son más densas y más asimétricas que aquellas ubicadas en las áreas laterales y posteriores; área que rodea al ápice frecuentemente erosionada. Las costillas son irregulares y abultadas con costillas secundarias finas en los espacios intercostales, las que a su vez se proyectan débilmente hacia el margen de la concha. Las líneas de crecimiento son prominentes y episódicas. El área apical de la concha es blanca y presenta manchas amarillas débiles e irregulares. El área intermedia varía de gris a azul hacia el margen que refleja el patrón externo de marcas oscuras y claras. Tamaño: 5-21 mm.

Anatomía: los tentáculos del manto son de tamaño similar. Presencia de cordón branquial.

Distribución: desde Caleta El Cobre, Chile $\left(24^{\circ} \mathrm{S}\right)$ hasta Pirulil, Isla de Chiloé, Chile $\left(42^{\circ} \mathrm{S}\right)$.

Ecología: habita sobre los discos y estipes de las algas: L. nigrescens en la zona norte y centro de Chile y $D$. antarctica en el sur de Chile. Comúnmente, se encuentra en la porción de los estipes ubicada cerca del disco, aunque también habita en los discos de fijación de ambas algas. Raramente se encuentra sobre rocas $\mathrm{u}$ otras algas. Solo en la latitud $29^{\circ} \mathrm{S}$ se registraron ejemplares presentes en los discos y estipes del alga $L$. trabeculata (Villouta \& Santelices 1986) en el intermareal inferior-submareal somero. Muñoz \& Santelices (1989) indican que los individuos de $S$. scurra de tamaño menor a $5 \mathrm{~mm}$ viven en pequeñas galerías excavadas en los discos del alga, mientras que los ejemplares de mayor tamaño se encuentran en cavidades desnudas y abiertas sobre los estipes. Las cavidades son horadadas en la parte proximal del estipe o antes de la primera bifurcación (Muñoz \& Santelices 1989). En estos casos, la concha de $S$. scurra aparece frecuentemente cubierta por algas del género Ulva. Ante la presencia de Heliaster helianthus, S. scurra no muestra respuesta activa de escape (C. Espoz observaciones personales).

Observaciones: este patelogastrópodo registra una de las reducciones más importantes en el tamaño del rango geográfico. En el pasado, su presencia fue descrita desde Tuquillo, Perú (10 S) (Talledo \& González 1989) hasta Tierra del Fuego, Chile (56º S) (Dell 1971), abarcando una distancia lineal de aproximadamente $5.000 \mathrm{~km}$. Sin embargo, el rango geográfico registrado a partir del presente estudio es de $2.066 \mathrm{~km}$. La reducción se registra en el límite norte de distribución y podría estar asociada con eventos de El Niño, ocurridos en el pasado (e.g., 1982-1983); esto debido a que dichos eventos están relacionados con un decrecimiento de las poblaciones del alga Lessonia nigrescens (Stanley 1984, Fuenzalida 1985, Castilla \& Camus 1992), principal hábitat de $S$. scurra. Adicionalmente, en la reducción del rango geográfico podría influir la fuerte presión de extracción a la que están siendo sometidas en la actualidad las poblaciones de L. nigrescens en la zona norte de Chile y sur del Perú (Castilla \& Camus 1992, C. Espoz observaciones personales).

\section{Scurria araucana (Orbigny, 1841) (Fig. 8b)}

Sinonimia: Patella araucana (Orbigny, 1841: 482); Collisella araucana (Dall, 1871: 257; Marincovich, 1973: 18; Alamo y Valdivieso, 1987: 15); Acmaea araucana (Pilsbry, 1891; Vol. 13, 35; Dall, 1909: 237); Collisella bahamondina (Ramírez, 1974: 19); Collisella canela (Ramírez, 1974: 21); Collisella lileana (Ramírez, 1974: 25); Collisella pullallina (Ramírez, 1974: 28); Collisella ruginosa (Ramírez, 1974: 28); Collisella lacerta (Ramírez, 1974: 24); Collisella pladilla (Ramírez, 1974: 28). 
Concha: perfil bajo a alto. Caracterizada por la presencia de costillas primarias marcadas que se proyectan más allá del margen de la concha, dándole a esta una apariencia crenulada. Internamente la concha es de color blanco brillante con un margen festoneado coloreado verde a café, alternándose con surcos blancos que se corresponden con las costillas externas. El área apical es coloreada, pudiendo ser verde, azul o café. En el área central se distingue la marca del miostraco, la cual varía entre café y gris. Tamaño: 6-22 mm.

Anatomía: los tentáculos del manto están alineados en una sola hilera y se alternan entre uno largo y uno corto de manera irregular. Estos tentáculos son más delgados que en $S$. ceciliana. La superficie oral está rodeada por una sola franja. Ausencia de cordón branquial.

Distribución: desde San Juan de Marcona, Perú ( $\left.15^{\circ} \mathrm{S}\right)$ hasta Pirulil, Isla de Chiloé, Chile $\left(42^{\circ} \mathrm{S}\right)$.

Ecología: presente en el intermareal rocoso superior a inferior en áreas expuestas y/o protegidas del oleaje. En plataformas horizontales y en pozas intermareales. Cuando habita en este último tipo de hábitat, $S$. araucana puede presentar una perfil de la concha bajo, erosionado, teñido de verde y similar a la apariencia externa de los cirripedios intermareales Jehlius cirratus (Darwin, 1854) y Notochthamalus scabrosus (Darwin, 1854). Comúnmente asociada a rocas cubiertas con una capa densa de algas. También en presenta sobre algas coralinas en la zona intermareal baja. Ante el contacto de Heliaster helianthus, S. araucana permanece inmóvil (Espoz \& Castilla 2000).

Observaciones: la especie presenta gran variabilidad en la morfología de la concha, la cual podría estar relacionada con la exposición al oleaje de los sustratos donde habita. La apariencia general, de la concha de esta especie se asemeja a la de Collisella scabra (Gould, 1846), presente en las costas de California.

\section{Scurria ceciliana (Orbigny, 1841) (Fig. 9A y 9B)}

Sinonimia: Patella ceciliana (Orbigny, 1841: 482); Acmaea ceciliana (Pilsbry, 1891: 33; Dall, 1909: 237; Theim, 1917: 617); Patelloida ceciliana (Powell, 1951: 80; Carcelles y Williamson, 1951: 258; Dell, 1971: 199); Collisella ceciliana (Marincovich, 1973: 19; Álamo y Valdivieso, 1987: 15); Collisella chaitena (Ramírez, 1974: 22); Collisella chilota (Ramírez 1974: 23); Collisella dalcahuina (Ramírez, 1974: 24); Collisella ortiguilla (Ramírez, 1974: 26); Collisella piteana (Ramírez, 1974: 27); Collisella silvana (Ramírez, 1974: 29); Collise- lla boehmita (Ramírez, 1974: 20); Collisella aconcaguina (Ramírez, 1974: 19).

Concha: la morfología de la concha es extremadamente variable y puede fluctuar desde especímenes relativamente planos y de superficie lisa hasta perfiles altamente cónicos con formas fuertemente marcadas por costillas. Los ejemplares de $S$. ceciliana frecuentemente cambian de sustratos durante su ontogenia, cambiando tanto el color como la morfología de la concha y su patrón de estriación. Esto hace que tanto el patrón morfológico como de coloración sean altamente flexibles. Dentro de los caracteres que se reconocen principalmente en individuos pequeños está una protoconcha de color café, concha clara con interespacios aún más claros y estriaciones muy finas e igualmente espaciadas. Con frecuencia estas estriaciones finas son visibles sobre las costillas y en los interespacios de la concha cerca del ápice. Con el crecimiento estas estriaciones se pierden, quedando solo las costillas en ejemplares de longitud mayor a $8 \mathrm{~mm}$. El número de costillas originadas tempranamente durante el crecimiento de la concha varía entre 10 y 18 en ejemplares de más de $10 \mathrm{~mm}$ de longitud. Las costillas frecuentemente son blancas, y aproximadamente del mismo tamaño que los espacios intercostales. Un análisis más detallado de la concha muestra que las costillas y los espacios entre ellas presentan diferentes proporciones de marcas claras y oscuras. Las marcas blancas toman la forma de triángulos, así como también de líneas radiales blancas. La superficie de las costillas tiene una concentración mayor de marcas que el espacio intercostal. Las costillas radiales blancas se concentran en la porción final de la parte anterior de la concha más que en la parte posterior. Las costillas no se proyectan en forma regular ya que muchas veces forman nodos a intervalos irregulares desde el ápice al margen lo que produce un margen suavemente crenulado. El color de la concha varía desde verde hasta gris. En estados adultos la concha frecuentemente aparece muy erosionada haciendo difícil el reconocimiento de las características mencionadas con anterioridad. Esta especie es susceptible a la infección del liquen Thelidium litorale (Espoz et al. 1995), y cuando esta ocurre solo el margen de la concha mantiene la morfología y color característicos de la especie (Espoz et al. 1995). Cuando hay infección, el ápice aparece fuertemente erosionado producto de lo cual la capa interna de la concha queda expuesta a la superficie externa. La capa interna de la concha es típicamente de color oscuro, variando desde enteramente negra a café donde la marca del miostraco es observable. También 
(A)
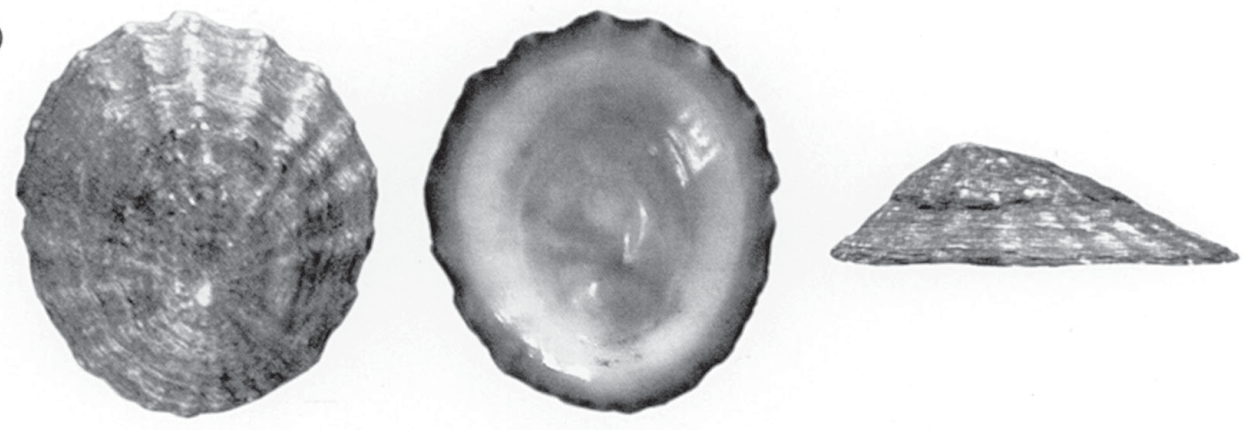

(B)
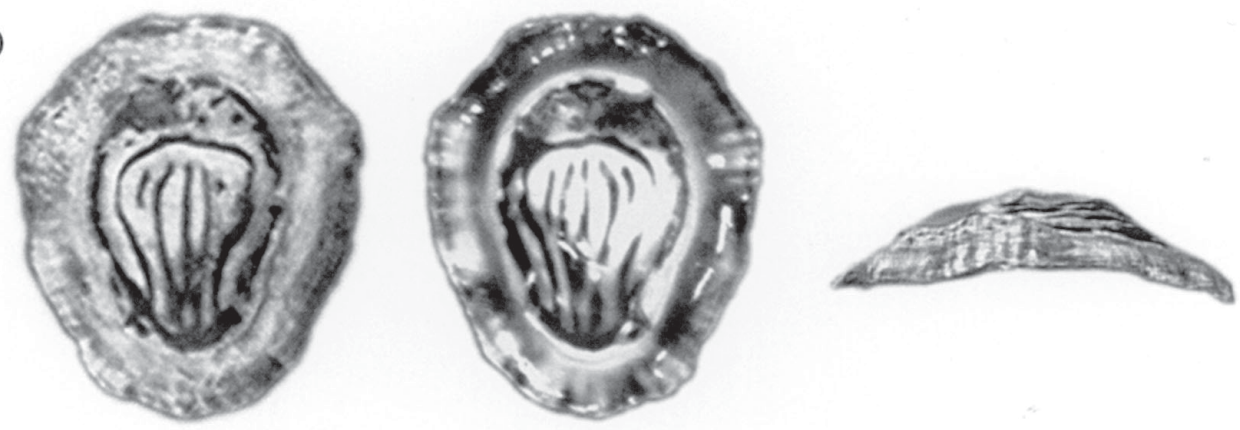

Fig. 9: Morfología de la concha. (A) Scurria ceciliana, longitud total 21,50 mm; (B) S. ceciliana, forma infectada por el liquen Thelidium litorale, longitud total 10,90 mm (ver Espoz et al. 1995).

Shell morphology. (A) Scurria ceciliana, total length $21.50 \mathrm{~mm}$; (B) S. ceciliana, infected by the lichen Thelidium litorale, total length $10.90 \mathrm{~mm}$ (see Espoz et al. 1995).

se pueden encontrar rayas verticales que se conectan en una dirección anterior-posterior (Espoz et al. 1995). Cuando las capas externas de la concha están erosionadas la coloración interna se hace visible en la superficie externa, incluyendo las líneas anterior-posterior. El miostraco puede o no estar remarcado por líneas finas oscuras. La parte intermedia entre el miostraco y el margen de la concha es típicamente blanco, siendo a veces de color azul. El margen de la concha presenta una alternancia entre marcas oscuras y claras reflejando el patrón externo de la concha. A veces, la presencia de costillas blancas es reflejada en el área intermedia. Tamaño: 6-18 mm.

Anatomía: los tentáculos del manto están ordenados en una hilera y son aproximadamente de igual tamaño. En comparación con S. araucana, la especie $S$. ceciliana presenta tentáculos más gruesos, de menor tamaño y más espaciados entre sí. La superficie oral de la boca es una franja simple. Ausencia de cordón branquial.

Distribución: desde Los Cangrejos, Perú (5 S) hasta Isla Navarino, Chile $\left(54^{\circ} \mathrm{S}\right)$.
Discusión: esta es una de las especies de patelogastrópodos de mayor variabilidad morfológica en el mundo (DR Lindberg observación personal).

Ecología: intermareal rocoso medio a superior en zonas expuestas y protegidas al oleaje. Plataformas verticales y horizontales, y también en pozas intermareales. Raramente se encuentra en la zona intermareal baja, excepto cuando existe una densa cobertura de algas. También habita sobre las conchas de Perumytilus purpuratus y Semimytilus algosus (Gould, 1850). Scurria ceciliana permanece estacionaria ante el contacto del asteroideo Heliaster helianthus (Espoz \& Castilla 2000). Los mismos autores indican que cuando el contacto es directo se registra un aumento de la adhesión contra el sustrato.

Observaciones: la especie se caracteriza por presentar un ecofenotipo que habita entre los cirripedios de la franja intermareal alta, el cual presenta altos niveles de infección por parte del liquen $T$. litorale. Este liquen cambia completamente el patrón externo de la concha, tanto en color como en morfología. El patrón resultante 
es una combinación de líneas café con puntos negros, no obstante algunas veces se observa enteramente café o gris (Fig. 7C, Espoz et al. 1995). Ocasionalmente, se presenta un color oscuro en la forma de reticulación o círculos concéntricos. Sumada a la modificación de color, la erosión de las capas de la concha por parte del liquen también modifica la escultura externa de la concha. Como resultado de estos factores, la superficie externa de la concha de S. ceciliana presenta un marcado parecido con la apertura de los cirripedios Jehlius cirratus y Notochthamalus scabrosus, los cuales habitan en el mismo nivel intermareal. Esta es la especie de patelogastrópodo endémico de la costa de Chile y Perú que presenta el rango geográfico más amplio con $3.360 \mathrm{~km}$.

\section{DISCUSIÓN}

En este estudio se entrega un sistema de clasificación para los patelogastrópodos intermareales endémicos de Chile y Perú, el cual refleja las relaciones de parentesco definidas a partir del análisis del gen 16S. Los estudios filogenéticos que incluyen a los patelogastrópodos de la costa del Pacífico sudeste son escasos (como excepciones ver Lindberg 1990, Simison 2000, Espoz 2002). Esto hace que la comparación con otros resultados dentro del área geográfica sea difícil. Sin embargo, la filogenia del grupo puede ser deducida a partir de los esquemas de clasificación propuestos por numerosos autores (ver referencias más adelante), suponiendo la monofilia de los taxa y la naturaleza sinapomórfica de los caracteres estudiados. Originalmente, las clasificaciones asignaron los patelogastrópodos endémicos de Chile y Perú al género Acmaea (Orbignyi 1841, Philippi 1846, Dall 1909, Dell 1971), siguiendo una tendencia mundial (Lindberg 1998). Esta asignación fue discutida por Lindberg (1981, 1986), quien concluyó que Acmaea es un género monotípico compuesto solo por $A$. mitra con parientes presentes en el submareal y no en la zona intermareal. Además de lo anterior, uno de los caracteres diagnósticos para los miembros de este género es la rádula. Así, de acuerdo con Lindberg (1981), Acmaea presenta una rádula con dientes laterales de igual forma y tamaño, sumado a la ausencia de dientes marginales o uncini. En los patelogastrópodos aquí estudiados, los dientes laterales son diferentes tanto en tamaño como en forma, así como también se presenta un par de dientes marginales. En la actualidad, se reconoce la presencia del género Acmaea solo para la costa noreste del Pacífico (Lindberg 1998).
Posteriormente, las clasificaciones ubicaron a los patelogastrópodos chileno-peruanos dentro de los géneros Scurria y/o Collisella (Marincovich 1973, Ramírez 1974, Paredes 1980, Talledo \& González 1984, Álamo \& Valdivieso 1987). Sin embargo, Marincovich (1973) reconoce que la asignación a uno u otro género es arbitraria y depende solo de si se considera o no el desarrollo de cordón branquial: Scurria presenta un cordón branquial desarrollado, mientras que Collisella no. Debido a la naturaleza variable de las estructuras branquiales (Lindberg \& McLean 1981, Lindberg 1988b), el género Collisella se reconoce hoy como sinónimo del género Lottia (Lindberg 1986, 1998), el que puede presentar o no cordón branquial desarrollado. En la actualidad, la estructura de la concha de los patelogastrópodos es considerada como un carácter más conservador debido a lo cual ha adquirido mayor relevancia cuando se trata de asignar los patelogastrópodos a uno u otro género. En este sentido, MacClintock (1967) encuentra que la estructura de la concha de los patelogastrópodos del litoral rocoso de Chile y Perú, pertenecientes al género Scurria, es diferente a la de otros patelogastrópodos registrados en el Pacífico este, especialmente a la de los patelogastrópodos del género Lottia, lo cual ha sido corroborado por otros autores (Lindberg 1981, 1986, Lindberg \& McLean 1981). Estructuralmente, la principal diferencia entre los mencionados grupos es la presencia en la concha de una capa externa prismática simple en Lottia, mientras que en Scurria la misma capa es del tipo prismática compleja (MacClintock 1967). Debido a lo anterior, en este estudio todas las especies endémicas al litoral rocoso de Chile y Perú, salvo ' $L$ '. orbignyi, fueron asignadas al género $\mathrm{Scu}$ rria. Este resultado es apoyado por los niveles de divergencia molecular entre secuencias que se registran para estos patelogastrópodos (Espoz 2002). En cuanto a las relaciones de parentesco, Lindberg (1990) plantea que la tribu Lottiini tiene como taxón hermano a la tribu Scurriini, cada una de las cuales incluye los clados formados por los géneros Lottia-Tectura y Scurria-Discurria respectivamente. Los resultados obtenidos a partir del análisis de la filogenia molecular realizado por Espoz (2002) corroboran una parte de la hipótesis de Lindberg (1990), puesto que apoyan la monofilia del grupo de patelogastrópodos del género $\mathrm{Scu}$ rria. Para el futuro, se plantea la necesidad de incluir el género monoespecífico Discurria en el análisis molecular, y hasta que ello no suceda, la división taxonómica planteada en el presente estudio resulta ser la más apropiada. 
A nivel intraespecífico, uno de los resultados más llamativos de este trabajo es la sinonimia propuesta para $S$. variabilis y $S$. parasitica. En su análisis de la región $16 \mathrm{~S}$ de ADN mitocondrial, Espoz (2002) determinó que ambas especies presentan secuencias moleculares idénticas, lo que explica la sinonimia de $S$. $p a$ rasitica con el nombre más antiguo de $S$. variabilis. De acuerdo con lo anterior, en el terreno la especie $S$. variabilis presentaría al menos tres ecofenotipos: (1) en sustratos rocosos a lo largo de todo su rango geográfico, (2) sobre las conchas de Fissurella crassa y F. limbata principalmente en Chile centro-sur y (3) sobre las placas del chitón Enoplochiton niger en el norte de Chile y sur del Perú. Adicionalmente, esta especie de patelogastrópodo presenta a lo menos dos formas transicionales, únicas en áreas geográficas específicas: (1) una registrada sobre sustratos rocosos en Pelancura $\left(33^{\circ} \mathrm{S}\right)$ y $(2)$ otra encontrada entre los $25^{\circ} \mathrm{S}$ y $28^{\circ} \mathrm{S}$ que vive sobre las placas de E. niger y sobre las conchas de $F$. limbata y $S$. viridula la cual fue molecularmente muy similar a las anteriores (ver Tabla $3)$. En función de estos resultados, resultaría especialmente interesante definir la estructura genética poblacional de $S$. variabilis considerando también la variación geográfica de los diferentes ecofenotipos y su posible relación con procesos de diversificación.

Otro resultado interesante fue el hallazgo de una nueva especie de patelogastrópodo, cercanamente emparentada a $S$. ceciliana. La diferencia molecular entre $S$. ceciliana y una de las formas registrada sobre las valvas del mitílido Perumytilus purpuratus (CP3 en Tabla 3 ) fue mayor a la supuesta para pares intraespecíficos. Debido a lo anterior se sugiere que esta forma sería una especie nueva. Asimismo, la diferencia molecular entre $S$. ceciliana y la nominada $S$. boehmita fue prácticamente nula por lo que ambas corresponderían a una sola especie (Tabla 3). Tomando en cuenta las fechas de descripción, la nominación definitiva correspondería a $S$. ceciliana. Evolutiva y ecológicamente, este resultado es llamativo puesto que plantea numerosas preguntas relacionadas con las variaciones morfológicas que se pueden presentar dentro de las especies de patelogastrópodos. Hockey et al. (1987) describe la ocurrencia de polimorfismo y mimesis críptica en una población de patelogastrópodos de $S$. variabilis en la costa de Chile central. Estos autores informan sobre tres morfotipos de $S$. variabilis y sugieren que la presión selectiva y diferencial por parte de aves depredadoras habría ayudado a mantener este polimorfismo. Posteriormente,
Espoz et al. (1995) indican que la asignación hacia $S$. variabilis por los mencionados autores, probablemente estuvo errada y que los ejemplares estudiados por Hockey et al. (1987) habrían correspondido a S. boehmita y $S$. ceciliana o $S$. araucana. Tomando en cuenta los resultados de Hockey et al. (1987), Espoz et al. (1995), Espoz (2002) y los de este estudio, es posible interpretar la variación morfológica de $S$. ceciliana como un polimorfismo donde una de las formas del genotipo de esta especie de patelogastrópodo presentaría una similitud importante con los cirripedios intermareales Jehlius cirratus y Notochthamalus scabrosus, la que es mediada por la acción del liquen Thelidium litorale (Espoz et al. 1995). El polimorfismo podría ser ventajoso para las poblaciones de patelogastrópodos cuando se consideran depredadores visuales como aves. En general, la selección natural favorecerá el desarrollo de características que hagan a los individuos presas menos conspicuos para sus depredadores (Sorensen \& Lindberg 1991). El camuflaje se puede obtener a través de la similitud con el ambiente físico inanimado (e.g., color del sustrato) o logrando una similitud con un organismo menos apetecido por el depredador. Entre los patelogastrópodos intermareales, los colores crípticos y las morfologías y esculturas variables aparecen como un fenómeno ampliamente distribuido. Por ejemplo, experimentos llevados a cabo por Lindberg \& Pearse (1990) en California mostraron que patelogastrópodos del género Lottia pueden cambiar facultativamente de color y de morfología de la concha para asimilarse al sustrato en el que habitan. Del mismo modo, en la costa de la isla de Vancouver, Columbia Británica, dos especies de Collisella dividen el microhábitat de acuerdo al color del sustrato logrando así una reducción de la depredación por peces y aves (Mercurio et al. 1985). Similarmente, Giesel (1970) sugiere que en el Pacífico noreste Aphriza virgata, ave migratoria común a lo largo de la costa de Chile (Castilla \& Paine 1987), es responsable de la selección estacional sobre formas del patelogastrópodo L. digitalis: organismos más claros y más obscuros que el fondo del microhábitat en el que habitan son más conspicuos a los depredadores. En Chile, la evidencia disponible sugiere que los patelogastrópodos del género Scurria son selectivamente depredados por aves siendo preferidos sobre los cirripedios. Específicamente, en el caso de las aves costeras A. virgata, Arenaria interpres y Haematopus ater (Castilla 1981, C. Espoz 
observaciones personales). Más aún, Hockey et al. (1987) presentan evidencias respecto de las preferencias de las aves Cinclodes nigrofumosus y $C$. patagonicus por las formas no crípticas de Scurria sobre la forma críptica similar a los cirripedios. Lo que hace más interesante este problema es que la forma críptica del genotipo de $S$. ceciliana aparece como el resultado de la relación entre el liquen $T$. litorale y la concha del patelogastrópodo (Espoz et al. 1995).

Finalmente, de las nueve especies de patelogastrópodos incluidas en el análisis molecular, 'Lottia' orbignyi fue la única que presentó distancias moleculares comparativamente mayores a las esperadas para pares interespecíficos (Tabla 3). Esta especie aparece filogenéticamente más relacionada con las especies del Pacífico noreste que con los patelogastrópodos miembros de la tribu Scurriini registrados en la costa rocosa de Chile y Perú (Simison 2000, Espoz 2002). Debido a esto, se sugiere una asignación preliminar al género Lottia. En este caso, el uso del género Lottia es provisional en espera de la secuenciación de todos los patelogastrópodos de la costa rocosa del Pacífico este, o bien de la secuenciación de otros genes que puedan dar luz respecto de la ubicación definitiva de la especie, tratada aquí, como 'Lottia' orbignyi.

\section{AGRADECIMIENTOS}

Agradecemos al Dr. Patricio Sánchez por el entusiasmo e interés que siempre mostró en relación con el tema de los patelogastrópodos. A A. Larrea, C. Santos, R. Espoz, A. Monares por el apoyo brindado en el terreno. En el Perú, el trabajo no hubiese sido posible sin la información entregada por el Dr. C. Paredes y el apoyo brindado por M. Rabi y C. Marabi. Asimismo, se agradece a quienes trabajan en el Instituto del Mar del Perú (IMARPE), en especial a E. Barriga, R. Mamani y O. Galindo. Diferentes personas colaboraron en nuestra revisión de colecciones sistemáticas. En el Museo de Historia Natural a P. Baeza, O. Gálvez y S. Letelier. En el Museo de Zoología de la Universidad de Concepción a L. Parra, V. Jerez y Minerva. Además, queremos agradecer en forma especial a P. Zavala de la ex Sala de Sistemática (PUC). A P. Marquet, J. M. Fariña, M. Méndez, B. López, R. Soto, J. Alvarado, S. Navarrete, M. Fernández y P. Camus por la discusión y comentarios entregados. C. E. agradece el financiamiento proporcionado a través de los proyectos FONDECYT No 2970076 y No 4990017.

\section{LITERATURA CITADA}

ALAMO V \& MV VALDIVIESO (1987) Lista sistemática de los moluscos marinos del Perú. Boletín del Instituto del Mar (Perú-Callao) Volumen Extraordinario: 1-205.

BRANCH GM (1985a) Limpets: evolution and adaptation. En: Trueman ER \& MR Clarke (eds) The Mollusca: 187-220. Evolution. Academic Press, London, United Kingdom.

BRANCH GM (1985b) Limpets: their role in littoral and sublittoral community dynamics. En: Moore PG \& R Seed (eds) The ecology of rocky coasts: 97-116. Hodder and Stoughton, London, United Kingdom.

BROOKS DR \& DA MCLENNAN (1991) Phylogeny, ecology, and behavior. A research program in comparative biology. University of Chicago Press, Chicago, Illinois, USA.

CARCELLES AR \& SI WILLIAMSON (1951) Catálogo de los moluscos marinos de la Provincia Magallánica. Revista del Instituto Nacional de Investigación de las Ciencias Naturales, Ciencias Zoológicas (Argentina) 2: 225-383.

CASTILLA JC (1981) Perspectivas de investigación en estructura y dinámica de comunidades intermareales rocosas de Chile central. II. Depredadores de alto nivel trófico. Medio Ambiente (Chile) 5: 190-215.

CASTILLA JC \& RT PAINE (1987) Predation and community organization on eastern Pacific, temperate zone, rocky intertidal shores. Revista Chilena de Historia Natural 60: 131-151.

CASTILLA JC \& PA CAMUS (1992) The Humboldt-El Niño scenario: coastal benthic resources and anthropogenic influences, with particular reference to the 1982/1983 ENSO. En: Payne AIL, KH Brink, KH Mann \& R Hilborn (eds) Benguela trophic functioning. South African Journal of Marine Science 12: 703-712.

DALL WH (1909) Report on a collection of shells from Peru, with a summary of littoral marine mollusca of the Peruvian zoological province. Proceedings of the United States National Museum 37: 147-294.

DELL RK (1971) The marine Mollusca of the Royal Society expedition to southern Chile, 1958-59. Records of the Dominion Museum (New Zealand) 7: $155-233$.

ESPOZ C (2002) Ecología y evolución de patelogastrópodos endémicos a la costa rocosa de Perú y Chile: distribución, abundancia y filogenia. Tesis doctoral, Departamento de Ecología, Facultad de Ciencias Biológicas, Pontificia Universidad Católica de Chile, Santiago, Chile. 252 pp.

ESPOZ C \& JC CASTILLA (2000) Escape responses of four Chilean intertidal limpets to seastars. Marine Biology 137: 887-892.

ESPOZ C, G GUZMÁN \& JC CASTILLA (1995) The lichen Thelidium litorale on shells of intertidal limpets: a case of lichen-mediated cryptic mimicry. Marine Ecology Progress Series 119: 191-197.

FUENZALIDA FR (1985) Aspectos oceanográficos y meteorológicos de El Niño 1982-1983 en la zona costera de Iquique. Investigaciones Pesqueras (Chile) 32: 47-52.

GIESEL JT (1970) On the maintenance of a shell pattern and behavior polymorphism in Acmaea digitalis. Evolution 24: 98-119.

GOLIKOV AN \& YI STAROBOGATOV (1975) Systematics of prosobranch gastropods. Malacologia 15: 185-232. 
GOULD AA (1846) Descriptions of the shells collected by the United States exploring expedition. Proceedings of the Boston Society of Natural History (USA) 2: 148-152.

GRAY JE (1840) Synopsis of the contents of the British Museum. Forty-second edition. London, United Kingdom. 370 pp.

GRAY JE (1847) A list of the genera of recent Mollusca, their synonymia and types. Proceedings of the Zoological Society of London for 1847: 129-219.

HOCKEY PA, AL BOSMAN \& PG RYAN (1987) The maintenance of polymorphism and cryptic mimesis in the limpet Scurria variabilis by two species of Cinclodes (Aves: Furnariinae) in central Chile. Veliger 30: 5-10.

HUPÉ LH (1854) Fauna chilena: moluscos. En: Gay C (ed) Historia física y política de Chile. Zoología 8. París, Francia. 499 pp.

KIMURA M (1980) A simple method for estimating evolutionary rate of base substitutions through comparative studies of nucleotide sequences. Journal of Molecular Evolution 16: 111-120.

KOUFOPANOU V, DG REID, SA RIDGWAY \& RH THOMAS (1999) A molecular phylogeny of the patellid limpets (Gastropoda: Patellidae) and its implications for the origin of their antitropical distribution. Molecular Phylogenetics and Evolution 11: 138-156.

LESSON RP (1830) Voyage autour du monde, sur la Corvette la Coquille, pendant les années 1822-1825. Voyage Coquille, Zoölogie 2. Paris, France.

LINDBERG DR (1981) Acmaeidae: Gastropoda, Mollusca. Boxwood Press, Pacific Grove, California, USA. $122 \mathrm{pp}$.

LINDBERG DR (1986) Name changes in the "Acmaeidae". Veliger 29: 142-148.

LINDBERG DR (1988a) Systematics of the Scurriini (new tribe) of the northeastern Pacific Ocean (Patellogastropoda: Lotiidae). Veliger 30: 387-394.

LINDBERG DR (1988b) The Patellogastropoda. Malacological Review (Supplement 4): 35-63.

LINDBERG DR (1990) Systematics of Potoacmaea fluviatilis (Blanford): a brackish water patellogastropod (Patelloidinae: Lottiidae). Journal of Molluscan Studies 56: 309-316.

LINDBERG DR (1991) Marine biotic interchange between the northern and southern hemispheres. Paleobiology 17: 308-324.

LINDBERG DR (1998) Order Patellogastropoda. En: Beesley PL, GJB Ross \& A Wells (eds) Mollusca: the southern synthesis: 639-652. Fauna of Australia. CSIRO, Publishing, Melbourne, Australia.

LINDBERG DR \& JH MCLEAN (1981) Tropical eastern Pacific limpets of the family Acmaeidae (Mollusca: Archaeogastropoda): generic criteria and descriptions of six new species from the mainland and the Galápagos Islands. Proceedings of the California Academy of Science 42: 323-339.

LINDBERG DR \& JS PEARSE (1990) Experimental manipulations of shell color and morphology of the limpets Lottia asmi and Lottia digitalis (Mollusca: Patellogastropoda). Journal of Experimental Marine Biology and Ecology 140: 173-185.

MACCLINTOCK C (1967) Shell structure of patelloid and bellerophontoid gastropods (Mollusca). Peabody Museum of Natural History, Yale University, New Haven, Connecticut, USA. Bulletin 22: $1-140$.

MADDISON WP, MJ DONOGHUE \& DR MADDISON (1984) Outgroup analysis and parsimony. Systematic Zoology 33: 83-103.
MANLY BFJ (1986) Randomization and regression methods for testing for associations with geographical, environmental and biological distances between populations. Research in Population Ecology 28: 201-218.

MARINCOVICH L Jr (1973) Intertidal mollusks of Iquique, Chile. Natural History Museum, Los Angeles County. Science Bulletin (USA) 16: 1-49.

MERCURIO KS, AR PALMER \& RB LOWELL (1985) Predator-mediated microhabitat partitioning by two species of visually cryptic, intertidal limpets. Ecology 66: 1417-1425.

MUÑOZ M \& B SANTELICES (1989) Determination of the distribution and abundance of the limpet Scurria scurra on the stipes of the kelp Lessonia nigrescens in central Chile. Marine Ecology Progress Series 54: 277-285.

ORBIGNY AD (1841) Voyage dans 1'Amerique méridionale. Mollusques (France) 5: 409-488.

PALUMBI SR (1996) Nucleic acids II: the polymerase chain reaction. En: Hillis DM, C Moritz \& BK Mable (eds) Molecular Systematics: 205-246. Sinauer Associates Inc, Sunderland, Massachusetts, USA.

PALUMBI SR, A MARTIN, S ROMANO, WO MCMILLAN, L STICE \& G GRABOWSKI (1991) The Simple Fool's Guide to PCR, Special Publication Department of Zoology, University of Hawaii, Honolulu, USA. $44 \mathrm{pp}$

PAREDES C (1974) El modelo de zonación en la orilla rocosa del departamento de Lima. Revista Peruana de Biología (Perú) 1: 168-191.

PAREDES C (1980) La familia Acmaeidae (Gastropoda, Archaeogastropoda) en el Perú. Revista Peruana de Biología (Perú) 2: 52-58.

PHILIPPI RA (1846) Diagnose einiger neuen ConchylienArten. Zeitschrift für Malakozoologie (Deutschland) 3: 19-21.

PILSBRY HA (1891) Manual of Conchology 13. Academy of Natural Sciences, Philadelphia, Pennsylvania, USA. 195 pp.

PONDER WO \& DR LINDBERG (1997) Towards a phylogeny of gastropod molluscs: an analysis using morphological characters. Zoological Journal of the Linnean Society 119: 83-265.

POWELL AWB (1973) The patellid limpets of the world (Patellidae). Indo-Pacific Mollusca (USA) 3: 75206.

RAMÍREZ J (1974) Nuevas especies chilenas de Lucapina, Fissurella y Collisella (Mollusca, Archaeogastropoda). Boletín del Museo Nacional de Historia Natural (Chile) 33: 15-34.

REEVE L (1843-1878) Conchologia iconica. London, United Kingdom. 20pp.

SIMISON WB (2000) Evolution and phylogeography of new world gastropod faunas. Ph.D. Thesis, Integrative Biology, University of California, Berkeley, USA. 214 pp.

SORENSEN FE \& DR LINDBERG (1991) Preferential predation by American black oystercatchers on transitional ecophenotypes of the limpet Lottia pelta (Rathke). Journal of Experimental Marine Biology and Ecology 154: 123-136.

SOWERBY GB (1839) Molluscous animals and their she1ls, by JE Gray, continued by GB Sowerby. En: Beechey FW (ed) The zoology of Capt. Beechey's voyage to the Pacific and Behring's Straits: 103155. London, United Kingdom.

STANLEY SM (1984) Marine mass extinctions: a dominant role for temperature. En: Nitecki MH (ed) Extinctions: 60-117. University of Chicago Press, Chicago, Illinois, USA. 
SWOFFORD DL (1999) PAUP: Phylogenetic Analysis Using Parsimony, Version 3.1. Illinois Natural History Survey, Champaign, Illinois, USA.

TALLEDO CR \& R GONZÁLEZ (1989) Moluscos gastrópodos y placóforos de la Provincia de Islay (Arequipa), Perú. Publicación Ocasional de la Universidad Nacional de San Agustín., Perú. 39 pp.

THIEM H (1917) Beitrage zur anatomie und philogenie der Docoglossen; II. Die anatomie und philogenie del Monobranchen (Akmaiden und Scurriiden nach der sammlung plates). Jenaische Zeitschrif für Naturwissenshaft (Alemania) 54: 405-603.
VERMEIJ GJ (1993) A natural history of shells. Princeton University Press, Princeton, New Jersey, USA

WILEY EO (1981) Phylogenetics: the principles and practice of phylogenetic systematics. Wiley J \& Sons (eds) New York, New York, USA. 158 pp.

WILEY EO, D SIEGEL-CAUSEY, DR BROOKS \& VA FUNK (1991) The compleat cladist: a primer of phylogenetic procedures. University of Kansas. Museum of Natural History. Special Publication 19. USA.

Editor Asociado: Patricio Camus

Recibido el 19 de agosto de 2002; aceptado el 21 de enero de 2004 